\title{
PROCEEDINGS OF THE THIRTY-THIRD ANNUAL MEETING OF THE AMERICAN SOCIETY FOR CLINICAL INVESTIGATION HELD IN ATLANTIC CITY, N. J., MAY 5, 1941
}

\author{
READ BEFORE THE SCIENTIFIC SESSION
}

The Relation of Sulfanilamide "Acidosis" to the Specific Inhibition of Carbonic Anhydrase. By W. BARRY Wood, JR. and Cutring B. Favour (introduced by $P$. H. Long), Baltimore, Md.

The cause of the "acidosis" which accompanies sulfanilamide therapy is not known. Mann and Keilin suggested a possible relationship between the acid-base disturbance and inhibition of carbonic anhydrase. This enzyme, contained in red blood cells, catalyzes the reaction $\mathrm{H}_{2} \mathrm{CO}_{3} \rightleftarrows \mathrm{CO}_{2}+\mathrm{H}_{2} \mathrm{O}$, and was shown to be specifically poisoned in vitro by unsubstituted sulfonamide derivatives $\left(\mathrm{R}-\mathrm{SO}_{2} \mathrm{NH}_{2}\right.$ ).

Using the manometric method of Meldrum and Roughton, we have extended the studies of Mann and Keilin and have made the following observations which suggest that sulfanilamide "acidosis" is due to inhibition of carbonic anhydrase:

1. Sulfanilamide added to blood in vitro $(10 \mathrm{mgm}$. per cent) reduced the enzymatic activity of the red cells to that of normal blood diluted 100 times. Sulfapyridine, sulfathiazole, and sulfadiazine did not affect the enzyme.

2. The bloods of all patients receiving sulfanilamide showed low $\mathrm{CO}_{2}$ combining powers and marked depression of carbonic anhydrase; the bloods of patients taking sulfapyridine, sulfathiazole, and sulfadiazine were normal.

3. By dialysis experiments in vitro the reaction between sulfanilamide and carbonic anhydrase was shown to be reversible.

4. When sulfanilamide was injected intravenously into dogs, inhibition of carbonic anhydrase and a marked fall in arterial $\mathrm{CO}_{2}$ content occurred within two minutes. The lowering of the $\mathrm{CO}_{2}$ content consistently followed inactivation of the enzyme.

Human Sulfathiazole Sensitivity. Observations upon the Febrile, Leukocytic and Immunologic Response. By Theodore J. Abernethy, Samuel C. Bukantz, and JoHn Minor (introduced by Theodore G. Klumpp), Washington, D. C.

Single doses of sulfonamide drugs were administered to a patient exhibiting fever, rash, and leukocytosis during treatment of lobar pneumonia with sulfathiazole. Two grams of sulfathiazole, on the 6th day of normal temperature following cessation of therapy, induced fever and marked leukocytosis. Appreciable amounts of the drug were detected in the blood 2 hours after its oral administration, but the febrile and leukocytic reactions were delayed for 4 hours. Other blood studies were normal. Similar doses of sulfanilamide and sulfamethylthiazole, although accompanied by identical blood concentration, induced no reaction, while response to sulfapyridine was minimal. That sensitivity had been retained during these negative responses was indicated by the persistence of re- sponse to as small a dose of sulfathiazole as 0.5 gram, given subsequently.

Skin tests of the sensitive patient, using saline solutions of the pure drugs, were negative. Attempts have been made to detect antibodies to coupled products of diazotized sulfathiazole and serum albumin, globulin, or resorcinol. Precipitation of these azo-antigens with the sensitized patient's serum, as well as certain unrelated immune sera, has been found to occur. Observations upon the specificity of this reaction and its application to the investigation of toxic manifestations to sulfonamides have been made.

Experimental and Clinical Studies on Gramicidin. By Wallace E. Herrell and Dorothy Heilman (introduced by Dr. B. T. Horton), Rochester, Minn.

A bactericidal substance isolated by Dubos (J. Exper. Med., 1939, 70, 1 ; Ann. Int. Med., 1940, 13, 2025) from a soil bacillus has a marked bactericidal action against gram-positive bacteria. This substance is toxic for laboratory animals when administered by the intravenous route. We have recently shown that one of the toxic effects of this substance is its hemolytic activity. The crude substance (tyrothricin) consists of two fractions, tyrocidine and gramicidin, as reported by Hotchkiss and Dubos (J. Biol. Chem., 1940, 136, 803). They found gramicidin to be the more active against the gram-positive bacteria.

Further studies in our laboratory have shown that the hemolytic effect of the crude substance is due to the presence of gramicidin. Using the tissue culture technic, we have determined the amounts of tyrocidine and gramicidin necessary to inhibit the growth of a number of strains of common gram-positive pathogenic bacteria. Small amounts of gramicidin ( 0.0005 to $0.0025 \mathrm{mgm}$.) inhibit the growth of all strains of pneumococci tested. Slightly larger amounts ( 0.005 to $0.01 \mathrm{mgm}$.) are required to inhibit strains of hemolytic streptococci, whereas still larger amounts are necessary to prevent growth of Streptococcus faecalis, Streptococcus viridans, and Staphylococcus. Tyrocidine is much less effective than gramicidin against all of these organisms. These results are drawn from approximately 2000 tissue culture preparations used in this study.

Clinical experiences with the local application of gramicidin in the treatment of infections caused by grampositive bacteria are reported at this time. Suitable methods of applying this substance locally are also reported.

Observations on the Use of "Gramicidin" (Dubos) in the Treatment of Streptococcal and Staphylococcal Infections. By Charles H. Rammelkamp (by invitation) and Chester S. Keefer, Boston, Mass.

Gramicidin is a bactericidal substance which was extracted from certain soil bacilli by René J. Dubos in 1939. 
It was capable of killing large numbers of gram-positive bacteria in vitro and it was found to be capable of protecting mice which had been injected with fatal doses of pneumococci.

The present report deals with the use of this substance in the treatment of hemolytic streptococcal and Staphylococcus aureus infections. The following facts emerge: (1) Relatively large amounts of gramicidin could be injected into the serous cavities of rabbits without producing any toxic effects. (2) Hemolytic streptococcal empyema in rabbits could be cured following the injection of the material into the pleural cavity. Dosage and the time elapsing between the onset of infection and treatment were important in the outcome. (3) Staphylococcus aureus infections in closed cavities were much more resistant to treatment and larger doses were necessary. (4) As far as the evidence goes, it seems clear that superficial infections in man due to staphylococci and streptococci respond in a satisfactory manner following the local application of the material. The dosage and the frequency of application require further study.

Penicillin as a Chemotherapeutic Agent. By Martin H. Dawson and (by invitation) Gladys L. HobBy, KARL Meyer and Eleanor Chaffee, New York, N. Y.

Fleming (1929) observed that a certain strain of penicillium produced a substance which exerted a marked antibacterial action. The substance was named penicillin. Chain et al (1940) reported that they had been able to obtain a considerable yield of penicillin. They showed that the substance exerted a remarkable antibacterial effect both in vitro and in vivo against gram-positive organisms, including anaerobes of the gas gangrene group.

Preparations of penicillin have been made in our laboratory which, in dilutions of one gamma per cc. inhibit the growth of 2,500,000 hemolytic streptococci. The effect appears to be bactericidal. Penicillin is also effective against the following organisms: pneumococcus, Streptococcus viridans, Staphylococcus, $\mathrm{Cl}$. welchii, $\mathrm{Cl}$. sporogenes and $\mathrm{Cl}$. septique. Penicillin is likewise effective in vivo. Mice can be protected against 100,000,000 lethal doses of hemolytic streptococci. In the dosage employed little or no toxic effect has been observed. The methods of preparation are described, together with such information as is known concerning its chemical nature. Brief mention is made of the use of penicillin in human infections.

\section{A Study of Sulfonamide Inhibitors by the Use of a Soil}

Bacillus Which Decomposes p-Aminobenzoic Acid. By

GeORGE S. Mirick (introduced by Colin M. MacLeod), New York, N. Y.

Substances inhibitory to the bacteriostatic action of the sulfonamide drugs are widely distributed in nature. $p$ aminobenzoic acid is an active inhibitor in quantities too small to be detected by the sensitive but non-specific diazo reaction. The great inhibitory activity of $p$-aminobenzoic acid raises the question of its identity with the other sulfonamide inhibitors.
A soil bacillus has been isolated which attacks $p$-aminobenzoic acid, destroying at the same time its diazo reaction and its activity as a sulfonamide inhibitor. The bacillus is quite specific, attacking both free and acetylated $p$-aminobenzoic acid, and $o$-aminobenzoic acid, but not $m$ aminobenzoic acid. It attacks novocaine but not the methyl and ethyl esters of $p$-aminobenzoic acid and none of the other related compounds tested except $p$-aminophenylacetic acid.

The bacillus has been used to study naturally occurring sulfonamide inhibitors. It destroys the inhibitory activity of yeast extract, which confirms other evidence that this activity is due to $p$-aminobenzoic acid. It does not inactivate the inhibitor present in peptone broth or a streptococcus extract. In fact, the bacillus itself forms or releases an inhibitor when grown in inhibitor-free liver infusion. The inhibitors present in broth and streptococci, and released by the bacillus in liver infusion, cannot be $p$-aminobenzoic acid since the microorganism destroys this compound.

The Relation of Ascorbic Acid to Human Complement. By Wesley W. Spink and (by invitation) Olaf Michelsen and Suzanne Agnew, Minneapolis, Minn.

It is stated that the titer of complement in guinea pig serum is quantitatively related to the amount of reduced ascorbic acid present. It is claimed that a deficiency of serum complement may be corrected by the in vivo or in vitro addition of reduced ascorbic acid. The application of this knowledge to human beings is still a matter of controversy. In view of the importance of complement in the immune mechanism, and the emphasis placed upon the ascorbic acid requirements in human infections, we have investigated the relationship between complement and ascorbic acid in man.

Twenty-three patients having little or no reduced ascorbic acid in their plasmas were given large doses of ascorbic acid intravenously. Ascorbic acid and complement titrations carried out simultaneously on blood specimens obtained before and immediately after the injections revealed no change in complement titer. The hemolytic action of human complement in serums deficient in ascorbic acid could not be increased by the in vitro addition of the vitamin. Complement and ascorbic acid were found to be unrelated by other means. The activity of complement was shown to be independent of the presence of reduced ascorbic acid by oxidizing the acid without changing the titer of complement. Complementary activity was abolished by chemical and physical methods without interfering with the reduced ascorbic acid.

Thus far, our investigations have failed to establish a relationship between the hemolytic action of complement and ascorbic acid.

The Effect of the Subcutaneous Injection of Histamine on the Histamine Content of the Blood of Patients With and Without Allergy. By BrAM Rose (by invitation) and J. S. L. BrownE, Montreal, Canada.

In previous studies it was noted that the symptoms of allergy may be associated with marked fluctuations of the 
histamine content of the blood, as compared to the marked stability of the blood histamine content of normal persons.

In order to investigate further the metabolism of histamine, the following procedure was carried out in 15 patients with allergic disease and in 15 non-allergic patients. The blood pressure, pulse and histamine content of the blood were first determined and again at five, fifteen and thirty minutes after the subcutaneous injection of $1 \mathrm{mgm}$. of histamine phosphate. Any other symptoms such as headache, flushing of the face, asthma or urticaria were also noted.

It was found that in cases of allergy, there was either no change, or a definite decrease in the blood histamine level, even when symptoms such as tachycardia, flushing of the face or headache were at their height. On the other hand, most of the non-allergic cases showed a definite increase in the blood histamine level when symptoms were pronounced, although a flat curve was also noted in several.

From these results it appears that the symptoms of histamine intoxication may be manifest in patients even though the histamine content of the blood remains at a stationary level or even decreases considerably.

The significance of these findings is discussed in relation to the changes in the histamine content of the blood in allergic disease and surgical shock.

Spectrophotometric Study of Acute Hemolytic Anemia During Sulfonamide Therapy with Detection of a New Blood Pigment-Methemalbumin. By CharLes L. Fox, JR. (by invitation) and Reuben OtTenberg, New York, N. Y.

The acute hemolytic anemia of sulfanilamide and sulfapyridine therapy was studied in four cases (two fatal) to determine the possible rôle of the abnormal blood pigments known to occur in this type of therapy.

Serum and urine containing the products of hemolysis and those erythrocytes which survived hemolysis were examined. Combined chemical methods and the objective Hardy recording spectrophotometer were utilized.

The red cells that survived hemolysis were essentially normal, containing but little methemoglobin. The urine contained very large amounts of hemoglobin and even larger amounts of methemoglobin up to 83 per cent. In the serum, representing the contents of the cells that underwent hemolysis, were found from 400 to $1700 \mathrm{mgm}$. per cent of three blood pigments: hemoglobin, methemoglobin and, contrary to expectation, not sulfhemoglobin but an entirely new pigment, methemalbumin.

This new pigment, recently discovered by Fairley, was prepared by incubating human hemoglobin with human serum. Its visible absorption spectrum (calculated on iron content) was obtained. This resembled methemoglobin except that in the red region a plateau-like maximum extended from $\lambda 610 \mathrm{~m} \mu$ to $\lambda 620 \mathrm{~m} \mu$.

Analysis of the results permits these conclusions:

1. Sulfhemoglobin played no rôle.
2. The cells that survived hemolysis were almost entirely free of methemoglobin, whereas the cells that underwent hemolysis apparently contained proportions of methemoglobin somewhat greater than occur in the cells of other patients who do not suffer acute hemolytic anemia.

3. After hemolysis there is a rapidly progressive conversion of the hemoglobin and methemoglobin in the serum to methemalbumin. This conversion to a pigment which is not excreted in the urine may account for the fact that only a small portion (not over one-tenth) of the liberated hemoglobin and methemoglobin appeared in the urine and may explain the "conservation" of blood pigments. Of the 500 to 700 grams of pigments liberated by the acute hemolysis, nine-tenths were rapidly removed from the circulation by extra renal mechanisms.

The Use of Purified Bovine Albumin Solutions as Plasma Substitutes.* By Charles A. Janeway and Paul B. Berson. (introduced by Soma Weiss), with the technical assistance of Mary A. Bradley, Anne Shwachman, and Thomas Thurston, Boston, Mass.

The method of fractionation of plasma by equilibration with ethanol-water mixtures recently reported by Cohn and his associates makes possible the large scale preparation of purified bovine plasma protein fractions. In collaboration with Professor Cohn and his group, we are carrying out clinical and immunological investigations on these preparations, particularly the albumin, because its properties of low viscosity and great stability, solubility, and osmotic activity render it a theoretically ideal substance for increasing and maintaining plasma volume in acute emergencies.

Bovine albumin and gamma globulin, each prepared as one fraction in this method, approach homogeneity as measured by electrophoresis and in the ultracentrifuge, and show very slight immunological relationship to one another. Cross-reactions between anti-beef albumin sera and the ordinary therapeutic horse sera are slight and diminish with increasing purity of the albumin fraction.

Beef albumin solutions will maintain blood volume in dogs with shock induced by burns, and very little of the injected albumin escapes in the urine. In man, the intravenous injection of such solutions in varying amounts has been carried out in 16 subjects to date without untoward reactions, except for serum disease in one subject who had previously had serum sickness from horse serum. Much more work must be done before beef albumin can be evaluated as a therapeutic agent in shock, but the investigations in progress indicate that it can be administered safely to man and that it is retained in the blood stream satisfactorily.

* Aided by a grant from the Proctor Fund of Harvard University and by a grant to Professor Edwin J. Cohn from the Administrative Committee of the National Research Council as recommended by the Executive Committee of the Committees Advisory to the Surgeons General. 
Changes in Osmotic Pressure of Erythrocytes During Storage. Rôle in Causation of Transfusion Reactions. By Elmer L. DeGowin and (by invitation) JoHn E. Harris, Joy Bell, and Robert C. Hardin, Iowa City, Ia.

The amount of hemolysis in blood kept at $2^{\circ} \mathrm{C}$. in the modified Rous-Turner dextrose-citrate preservative for 30 days was approximately one-half that occurring in citrated blood during 10 days of storage. Maximum inhibition of hemolysis during refrigeration required a concentration of at least 3 per cent dextrose in the blood mixture. The cell volume and the erythrocyte fragility gradually increased during the storage of citrated blood. When the blood-dextrose-citrate mixture was collected, the cell volume increased about 50 per cent with a corresponding change in fragility. This was due to diffusion of dextrose and water into the cells. The sugar could be washed out by physiologic saline, and normal fragility values could be obtained. There was an almost equivalent exchange between plasma sodium and cell potassium during storage but neither this nor the diffusion of chlorides, inorganic phosphorus, and lactic acid, nor $\mathrm{pH}$ changes could account for the secondary swelling.

When a 10 per cent dextrose solution was substituted for the 5.4 per cent concentration in the blood-dextrosecitrate mixture, the initial swelling of the erythrocytes was obviated. During storage the cell contents became so hypertonic that, when transfused, they were hemolyzed by the serum of the recipient. Sixteen hemolytic transfusion reactions were thus incurred. The excessive increase in osmotically active cell contents during preservation constitutes another criterion governing the limitation of storage. Over 2500 transfusions of blood stored in the 5.4 per cent dextrose-citrate solution have been administered. The storage limit of 30 days has proved satisfactory.

Recent Advances in Clinical Medicine with the Aid of Artificially Prepared Radioactive Isotopes. Вy ЈонN H. LAWrence and (by invitation) Joseph G. Hamiton, L. A. ERf, and Charles Pecher, Berkeley, Calif.

Radio-phosphorus has been shown to be selectively accumulated in the tissue infiltrated with leukemic cells of leukemic mice. The selective deposition of this radioactive substance in these tissues suggested that radiophosphorus might be of therapeutic value in human leukemia since it appeared likely that the regions which had become infiltrated by leukemic cells would contain a relatively higher proportion of the administered radiophosphorus than the uninvolved tissues, and thus be subjected to a selective irradiation from the accumulated radio-phosphorus. More than 100 patients with chronic lymphatic and myelogenous leukemia have undergone therapy with radio-phosphorus during the past three years. Out of this group, 30 patients had received no other form of therapy prior to the use of the radio-phosphorus. One of the patients in this second group has had a complete clinical and hemotological remission for two years, 2 for one year and 16 have had clinical remissions for 6 months or longer. A sufficient period of time for a thorough evaluation of this new type of therapy has not as yet elapsed, although it appears that more favorable results are to be expected than have been encountered with the conventional methods of treatment of the chronic leukemias. The complete absence of radiation sickness has been consistently observed in all of the patients treated with radio-phosphorus and no other toxic manifestations have been encountered with the therapeutic use of this material. The acute forms of lymphatic and myelogenous leukemia have responded no better to radiophosphorus than to pre-existing therapeutic methods, such as $\mathrm{x}$-ray, radium, etc. Encouraging results have been noted in several cases of polycythemia vera which have received radio-phosphorus.

Animal experimentation has demonstrated that radiostrontium is deposited in bone with a high degree of selectivity. In view of this observation, radio-strontium has been employed in the treatment of several patients suffering from generalized carcinomatosis which was characterized by widespread metastases in the skeleton. As yet no positive roentgenological evidence of regression of the bony metastases has been observed but some of the patients have had a considerable degree of relief from pain.

Radio-iodine has been employed in the study of the metabolism of iodine by the thyroid glands of normal human controls and patients suffering from several different types of thyroid disorders. It has been observed that, with the aid of radio-iodine, the thyroid glands of these different clinical entities handle iodine in a characteristic manner. It has also been shown that it is possible to completely destroy normal thyroid glands in animals by the administration of radio-iodine without producing demonstrable damage to the other tissues of the body.

\section{A Study of the Selective Absorption of Iron With the} Aid of its Radio-Active Isotope. By CARL V. Moore, Harold Roberts and Virginia Minnich (introduced by D. P. Barr), St. Louis, Mo.

The work of Hahn, Whipple and their associates has provided convincing proof that iron-deficient dogs absorb larger quantities of iron than do normal animals. While there is considerable evidence that this is also true for man, it has been demonstrated repeatedly, both by balance studies and by the serum iron absorption curve technic, that the normal human adult absorbs relatively large quantities when he is given therapeutic doses of various iron salts. In order to clarify this apparent discrepancy, we have re-studied the absorption of iron both in dogs and in human subjects, using the serum iron absorption technic and the method of Hahn and Whipple which requires the radioactive isotope of iron.

It has been possible to confirm the fact that irondeficient dogs absorb iron well, while normal animals show only minimal evidence of iron absorption. Normal dogs, however, who have been subjected to one massive hemorrhage seven days before receiving iron, absorb 
quantities which closely approximate those absorbed by anemic animals. If an animal whose iron reserves have been exhausted by repeated phlebotomy receives in one massive injection sufficient colloidal ferric hydroxide to replace all of the removed iron, and is studied immediately after the replacement, it is found that he absorbs the metal apparently as well as if there had been no replacement. However, if seven or eight days are permitted to intervene between the time of iron replacement and the absorption study, then only minimal amounts of iron are absorbed. It has been demonstrated that the level of iron in the blood plasma is not the controlling factor which determines the extent of absorption.

In contrast with the studies outlined above, we have continued to obtain evidence that the normal human subject absorbs large quantities of iron when therapeutic doses are given, even though precautions are taken to assure the adequacy of iron reserves.

The Selective Absorption of Radioactive Iron by Normal and Iron-Deficient Hunian Subjects. By JoSEPH F. Ross and Milan A. Chapin (introduced by James M. Faulkner), Boston, Mass.

Investigations of iron metabolism with the artificially radioactive isotope, $\mathrm{Fe}^{\infty}$, have been extended to human subjects. Adults with normal blood and presumably adequate reserves of iron absorbed only very small quantities of the isotope. By contrast, patients with hypochromic anemia and presumably depleted reserves of iron assimilated large amounts of the element.

Negligible amounts of iron were absorbed by patients with untreated pernicious anemia who, presumably, had adequate reserves of iron, even though the anemia was very severe.

Large quantities of iron were absorbed by these same patients, however, after their iron stores had been depleted by producing active hemoglobin regeneration with liver extract therapy, by performing phlebotomies frequently, and by restricting the iron intake. This absorption occurred even though the anemia had abated and was of mild degree.

It would appear, therefore, that the reserves of iron in the body, and not the degree of anemia, determine whether or not iron is absorbed. It is possible that such selective absorption of iron is controlled by the amount of tissue iron present locally in the tissue of the gastro-intestinal mucosa.

The Effect of Foreign Surfaces on Blood Coagulation. By Eugene L. LozNer and F. H. L. TAYLOR (introduced by George R. Minot), Boston, Mass.

The effects on blood coagulation of glass, paraffin, collodion and a plastic (Lusteroid) were investigated. The platelet content of normal citrated plasma was varied by centrifuging at different speeds and the plasma was then placed in tubes of the four materials. Both the coagulation time of the plasmas on recalcification, and the clotpromoting activity of the plasmas for hemophilic blood, were found to be essentially independent of the platelet count. Of the various foreign surfaces investigated, glass appeared to exercise the greatest effect on shortening the coagulation time when the plasma was recalcified. The clot-promoting activity for hemophilic blood of normal plasma protected from a glass interface, and of the acidprecipitated globulin fraction derived from such plasma, was considerably less than that of similar preparations exposed to glass. It is concluded that certain foreign surfaces modify blood coagulation by their effects on the factor in normal cell-free plasma concerned with its clot promoting activity for hemophilic blood rather than by their effects on platelet destruction. The implications of these findings to the blood coagulation reaction are discussed.

Metabolic Studies of the Utilization of Extra Dextrose by Patients with Controlled Diabetes Mellitus. By James A. Greene and (by invitation) ANN David, Iowa City, Ia.

Patients with controlled diabetes mellitus have been shown to utilize extra dextrose without an increase of insulin dosage. The mechanism of this utilization has been studied in 8 patients. There have been 27 observations of 4-hour periods in a respiratory chamber during which the gaseous and nitrogen metabolism has been studied. The utilization of the dextrose of the diet was determined first. A few days later the utilization of the extra dextrose was ascertained following the ingestion of the diet plus 50 grams of dextrose. In 4 cases the extra dextrose was administered for from 4 to 6 days, when the second study was repeated. In 4 other cases the utilization of the dextrose equivalent of the diet was also ascertained and a few days later the utilization of the dextrose equivalent of the diet plus 50 grams of extra dextrose was determined. Very little of the extra dextrose was oxidized. Comparison of the non-protein respiratory quotients indicates that it was stored as glycogen. Only one respiratory quotient was above 1.00 . A mild insulin reaction in 2 cases reduced the dextrose oxidized.

The significance of these observations upon the treatment of diabetes mellitus is discussed.

The Influence of Insulin on the Respiration of Human Diabetic Muscle in Vitro. By Henry T. RicketTs and F. J. Stare (introduced by C. Phillip Miller), Chicago, Ill.

The respiration of human diabetic muscle was studied by the Warburg technic in a glucose Ringer phosphate buffer with and without the addition of insulin. In muscle taken from patients with severe, poorly controlled, " insulin sensitive" diabetes, insulin alone, or in one case only after the addition of fumarate, caused an increase of about 40 per cent in the respiration. This effect was not obtained in the muscle of "insulin resistant" diabetics (including two patients with coexisting acromegaly) or of non-diabetic individuals; nor was it observed in any case when pyruvate was substituted for glucose in the buffer. 
In the case of one patient with severe diabetes, the oxygen uptake of the muscle removed prior to any treatment was definitely increased by the addition of insulin to the Warburg chamber, whereas no augmentation occurred in tissue obtained after the disease had been adequately controlled with insulin or in a third experiment performed several months later when the patient had become highly resistant to insulin, requiring from 500 to 3000 units per day.

This is the first direct evidence of which we are aware that insulin has an effect on the respiration of human diabetic muscle.

Factors Influencing Renal Excretion of Calcium in the Normal and in the Hyperthyroid State. By T. L. AlTHAUSEN and (by invitation) M. STock holM and W. J. KerR, San Francisco, Calif.

Urinary excretion of calcium was studied in rats from the following aspects: (1) the effect of unrestricted, decreased, and increased water intake; (2) the influence of variations in dietary calcium; and (3) the effect of variations in diuresis produced by xanthin diuretics. These experiments were performed in normal and in hyperthyroid rats.

Our data show that in normal rats the urinary excretion of calcium is directly proportional to the intake of water, to the dietary calcium, and to the degree of diuresis.

Administration of thyroxin resulted in the customary rise of urinary calcium which paradoxically takes place in hyperthyroidism without hypercalcemia. Since our hyperthyroid rats approximately doubled their water intake and ingestion of food (thereby receiving more calcium), and since thyroxin produces diuresis, it appears that these three factors explain the increased urinary calcium output in hyperthyroidism. Conversely, a decrease in water intake or in dietary calcium diminished the urinary calcium of hyperthyroid rats to normal levels, thus further supporting this conclusion.

The bearing of this work and of our previous study of the calcium exchange of the intestine on various hypotheses explaining the negative calcium balance in hyperthyroidism is discussed. Our studies suggest that therapeutic administration of calcium should restore the calcium balance to normal.

\section{A Renal Concentration Test Employing Posterior Pitui-} tary Extracts. By Wm. A. Sodeman and (by invitation) H. T. ENGELHARDT, New Orleans, La.

We have abolished the long periods of water deprivation necessary in renal concentration tests by the use of the antidiuretic action of posterior pituitary extracts. Unprepared patients were given $0.5 \mathrm{cc}$. (10 units) surgical pituitrin and specimens collected at half-hour intervals. Specific gravity was determined with a pyknometer. This procedure was compared with a standard concentration test (modified Fishberg test), the standard concentration test plus pituitary extract, and with the administration of $1600 \mathrm{cc}$. of water with and without pituitary extract.
In 97 tests on 30 individuals results have been consistent. Posterior pituitary extract in unprepared patients invariably elevated the specific gravity to values which equalled or exceeded those obtained with water deprivation. Water diuresis $(1600 \mathrm{cc}$.) was inhibited by the pituitary extract with maintenance of low urinary volume and elevation of the urinary specific gravity. Results were similar in both normal and abnormal subjects.

Pregnancy and angina pectoris are contraindications to the test. Hypertension is not. The dosage of pituitary extract used has not influenced the blood pressure.

This test eliminates undesirable and unnecessary periods of water restriction. It may be used at any time of day on unprepared patients.

Renal Blood Flow, Filtration Rate and Tubular Excretory Mass in Patients with Specific Toxemia of Pregnancy. By Catherine A. Welsh, Irwin Wellen and Howard C. Taylor, JR. (introduced by James A. Shannon), New York, N. Y.

This report concerns the measurement of renal blood flow (diodrast clearance), filtration rate (inulin clearance), tubular excretory mass (diodrast $T_{m}$ ) and phenol red clearance in patients with specific toxemia of pregnancy. These observations were made on thirteen patients in fourteen pregnancies, both antepartum and postpartum, and in most instances on several occasions.

The patients were seen early in pregnancy when they exhibited no evidence of vascular or renal disease and no hypertension. In the last trimester, hypertension, proteinuria and edema appeared in all. In seven, there was clinical cure after delivery, while in six, hypertension has persisted.

We have studied eight normal pregnant women by the same methods. The results show that there is no change in renal blood flow or filtration rate in normal pregnancy.

Before delivery the patients with specific toxemia showed a slight reduction in filtration rate, a renal blood flow of normal value or distinctly above normal in some instances, and a somewhat reduced filtration fraction.

After delivery, the filtration fraction increased in all; it became normal in the cured group and it rose to above normal in the hypertensive group, in part because of an increase in filtration rate, and in part because of a decrease in blood flow. The fall in blood flow was greater in the hypertensive group. The postpartum findings in the cured group are within normal limits, while the postpartum findings in the hypertensive group present the picture of efferent arteriolar spasm and renal ischemia such as that typically seen in essential hypertension.

The postpartum fall in renal blood flow and increase in filtration fraction were found in the first observation after delivery, regardless of whether the blood pressure had fallen to normal or not. This demonstrates that renal blood flow in these individuals is not determined solely by mean arterial pressure and must be related to renal vascular changes.

In respect to the genesis of hypertension in both the cured and hypertensive groups, it may be emphasized that 
these groups present a picture of sufficiency of renal blood flow, or actual renal hyperemia. Thus we do not believe that renal ischemia is primarily responsible for hypertension in toxemia of pregnancy.

\section{A Comparison of Pituitrin and the Antidiuretic Substance} in Human Urine and Placenta. By George C. HAM* (introduced by Eugene M. Landis), Charlottesville, Va.

The presence of an antidiuretic substance in the urine of patients with the toxemias of pregnancy (Teel and Reid, Endocrinology, 1939, 24, 297) has been confirmed. On the basis of Gilman and Goodman's results in rats (J. Physiol., 1937, 90, 113), it has been assumed widely that this substance has its origin in the pituitary, but Walker (Am. J. Physiol., 1939, 127, 519) and Arnold (Arch. f. Exper. Path. u. Pharmakol., 1938, 190, 360) found that hypophysectomy did not affect the antidiuretic activity of the urine in animals.

Because of this uncertainty, the antidiuretic substance of human and rat urine has been compared with commercial pituitrin and pituitary extracts with respect to dialysis through cellophane, ultracentrifugation, and urinary chloride excretion. Notable differences between pituitrin and the antidiuretic substance of urine were observed. Placental extracts were found to have antidiuretic activity which closely resembled that of the urine concentrates from the same patients.

The rat method of Burn (Quart. J. Pharm. and Pharmacol., 1931, 4, 517) was used with certain modifications. With uniform water loads, by gavage, the solutions to be tested were injected intraperitoneally and the urine volumes of control and experimental groups were plotted against time. The areas of these curves were measured with a planimeter and the grade of antidiuresis was expressed in square inches.

Ultracentrifugation. Samples of potent human urines were ultracentrifuged at 60,000 r.p.m. for 3 to 6 hours, divided into four fractions, and assayed for antidiuretic activity. In every case the fourth or bottom fraction showed the greatest activity. Conversely, varying dilutions of pituitrin solutions ultracentrifuged in the same manner showed no constant difference between the four fractions. The active principle of placental extracts, like the urine substance, was concentrated by ultracentrifugation.

Dialysis. The urine and placental antidiuretic factors are not dialyzable through cellophane, whereas the pituitrin factor is dialyzable.

Chloride excretion. It is well known that pituitrin increases the urinary chloride excretion and this was confirmed. The antidiuretic substance in urine and placenta did not increase urinary chloride excretion.

Crude extract. Commercial pituitrin is a hydrolytic product of the posterior pituitary gland and may therefore consist of a smaller molecule than the native hormone, and thus fail to concentrate in the ultracentrifuge. Crude, saline extracts of fresh, frozen lobes of the posterior pituitary gland were found to be definitely anti-

\footnotetext{
* Commonwealth Fund Fellow.
}

diuretic and to increase urinary chloride excretion markedly. The antidiuretic factor and the factor which increases urinary chloride excretion were both dialyzable through cellophane and they were not concentrated by the ultracentrifuge. Further work with absolutely fresh press-juice is under way.

Kidney extracts. Preliminary work shows that certain concentrations of heated kidney extract produce moderate antidiuresis, while other concentrations are conspicuously diuretic. Both weak and concentrated solutions increase the excretion of chlorides markedly, presumably due to their renin content. Further study of these two effects is being carried on.

\section{Conclusions}

1. The antidiuretic substance in human and rat urine has different physical and biological properties from the antidiuretic factor of pituitrin.

2. The antidiuretic factor in placental extracts has in three respects, at least, the same physical and biological properties as the urine factor.

Prostate and Serum "Acid" Phosphatase. By AlexANDER B. Gutman and (by invitation) Ethel B. GutMAN, New York, N. Y.

Further studies have been made of (1) the physiological significance of the specific "acid" phosphatase present in high concentration in human prostate tissue, (2) of the usefulness of serum "acid" phosphatase determinations in the diagnosis of metastasizing prostate carcinoma.

It has been found that " acid" phosphatase first appears in the prostate gland at puberty, either normally or artificially induced; that the enzyme content of seminal fluid is extremely high, relatively constant for any one individual, and largely contained in the first cc. of ejaculate; that the enzyme may form part of an extracellular glycolytic system (analogous to intracellular erythrocyte phosphatase), participating in the largely glycolytic metabolism of human spermatozoa.

Invading prostate carcinomatous tissue secretes " acid" phosphatase into the circulating fluids where the enzyme can be determined and the primary tumor identified. Our experience with 62 cases of metastasizing prostate carcinoma, in 37 cases of prostate carcinoma without demonstrable metastases and in several hundred miscellaneous cases indicates that the serum " acid" phosphatase determination is useful in detecting metastasizing prostate carcinoma, and occasionally before this is possible by $\mathbf{x}$-rays. Paget's disease and other osteoplastic bone lesions can be differentiated.

Nitrogen Balance in Cirrhosis of the Liver. By JoSEPH Post (by invitation) and Arthur J. Patek, Jr., New York, N. Y.

Nitrogen balance studies were performed on 5 patients with cirrhosis of the liver, ascites, and hypoalbuminemia in order to determine whether the hypoalbuminemia was due to altered protein synthesis or to protein starvation caused either by low protein intake or by faulty assimila- 
tion. Abdominal paracenteses were not done during the study periods. In 3 patients the nitrogen balance was determined for 48-hour periods at 2-week intervals for 1, 2, and 2 months, respectively. Two other patients were studied for 25 and 32 consecutive days. The patients were fed daily 1.5 to 2.0 grams of protein per kilo body weight.

The results show that the fecal nitrogen was within normal limits. All patients were in positive nitrogen balance. No correlated rise of serum albumin was associated with this nitrogen retention. The serum globulin and nonprotein nitrogen were unchanged. This type of balance pattern differs from that seen in nutritional hypoproteinemia, in which a rapid rise of serum albumin follows protein feeding. The findings indicate that patients with cirrhosis of the liver, ascites, and hypoalbuminemia can absorb and retain nitrogen from the food protein. However, there appears to be a defect in the synthesis of serum albumin.

\section{Dietary Liver Disease in Rats. By Graham Webster}

(introduced by Joseph T. Wearn), Cleveland, Ohio.

Rats given a diet of 8 per cent casein, 38 per cent fat, 2 per cent cod liver oil, 5 per cent salts and 47 per cent rice starch with 20 gamma each of thiamin chloride and riboflavin or Brewer's yeast $1 / 2$ gram q.o.d. developed a disease of the liver consisting of portal cirrhosis with occasional necrosis at the end of a 150-day period. They also exhibited nephrosis and cortical necrosis of the kidneys. These lesions could be prevented by increasing casein to 18 per cent, by the addition of betaine $50 \mathrm{mgm}$. q.d., and by diminishing fat. The addition of cystine $5 \mathrm{mgm}$. q.d. and of cholesterol 2 per cent aggravated the disease and could be inhibited by the addition of betaine. Whole yeast 1 gram daily or molasses 2 grams daily, added to the original diet in place of some carbohydrate, prevented the lesions. Male rats of more than $250 \mathrm{gram}$ weight were most susceptible to liver changes. Kidney changes occurred at any age. In the rats receiving added cystine there was an incidence of neoplasms in 20 per cent; 2 hepatomas, 1 carcinoma of the lung, 1 adenocarcinoma of the pancreas and 1 unidentified retroperitoneal tumor. No tumors occurred in any of the other rats used in the experiment.

Studies on Blood Flow in the Gastro-intestinal Tract of Man. By C. H. Richards and Stewart Wolf (by invitation) and H. G. WolfF, New York, N. Y.

A method of measuring and recording changes in the blood flow of the intestinal mucosa has been developed. The essential element consists of a silver button, which carries a heater and a thermocouple, mounted on the surface of a balloon. The button is applied to the mucosa of the gut by inflating the balloon, and the temperature of the button is raised about $2^{\circ} \mathrm{C}$. above body temperature by connecting the heater to a suitable D.C. source. The reference thermocouple is maintained at body temperature. With constant heat supplied, the temperature of the button will be a function of the rate at which heat is conducted away by the blood flowing past it.
Blood flow (temperature) is recorded by connecting the thermocouples to a galvanometer, the light reflected from its mirror varying the illumination of a photoelectric cell. The current from this cell is then amplified and fed into a solenoid whose movable core carries a writing point.

Pressure is recorded simultaneously by a similar solenoid and amplifier controlled by the height of an $\mathrm{Hg}$ column in contact with a high resistance wire.

It has been shown by this method that contractions in the duodenum, ileum and colon are usually associated with a transient increase in mucosal blood flow. In fasting subjects the sight and smell of food were accompanied by a marked increase in flow in the duodenal mucosa. Experimentally induced emotional states, e.g., anxiety and resentment, may also augment the circulation in the duodenum.

On the Mechanism of Insulin Resistance in Toxemic States. By Matthew Taubenhaus (by invitation) and Samurz Soskin, Chicago, Ill.

Previous work has clearly shown that the liver is a major factor in determining carbohydrate tolerance and the reaction of the whole animal to insulin. The study of the hepatic enzyme systems involved in these processes, and of the influence of toxin upon them, necessitates the frequent sampling of the liver for biological assay. The present work was therefore done on normal dogs and on animals treated with diphtheria toxin.

It is known that hepatic glycogenolysis normally proceeds by phosphorylosis, and that insulin acts upon this system, although the exact point of action is still in doubt. The present work shows that toxemic hepatic damage inhibits the phosphorylating systems, and at the same time allows the blood amylase, which is normally excluded from (or inactive within) the liver cell, to enter and break down glycogen. Insulin does not influence this latter abnormal pathway of glycogenolysis and hence an abnormally small response to insulin is exhibited. This work also supplies a rationale for high carbohydrate therapy in insulin resistance on the basis of mass action on the amylolytic enzymes.

The Excretion of Zinc Uroporphyrin in Idiopathic Porphyria. By C. J. Watson and (by invitation) Samuel Schwartz.

Five cases of idiopathic porphyria, either abdominal, nervous, or mixed in type, have been studied during the past three years. Three of the five cases died and were examined at necropsy; two are living and have improved to some extent.

All of the cases have been characterized by the excretion of uroporphyrin in both urine and feces in the form of the zinc complex. The occurrence of uroporphyrin in the feces has hitherto not been described. The excretion of uroporphyrin as the zinc complex in five successive cases of this disease indicates that zinc uroporphyria is a disease entity, and that excretion of uroporphyrin as the zinc complex is the usual rather than the unusual mode of elimination in so-called "acute" idio- 
pathic porphyria. The site of formation of uroporphyrin and the significance of its combination with zinc have not been determined.

Except in one instance, neither free uroporphyrin nor its zinc complex has been found in the bile, gall stones, or duodenal contents. Zinc uroporphyrin was identified with certainty in the duodenal contents of one of the cases. The amount appeared to be relatively small in comparison with that noted in the urine and feces of the same case. The significance of this absence or relative lack of uroporphyrin in the bile, as compared with the urine and feces, is not yet clear. Two possibilities may be considered: (1) that the substance is formed in the bowel and deposited in part in the liver (where it has been found in large amounts at necropsy), another part passing into the general circulation and thence into the urine; (2) that the substance is formed in the body and excreted through the intestinal mucosa. This possibility is considered chiefly because of the known intestinal excretion of zinc.

In addition to the zinc complex of uroporphyrin, the urines of these patients regularly exhibited positive Ehrlich reactions, due chiefly to porphobilinogen. A simple method serving to distinguish this substance from urobilinogen is described. This depends upon chloroform solubility. The Ehrlich compound of urobilinogen is readily extracted with chloroform while that of porphobilinogen is not.

Physiological Effects of Reduction of Internal Temperature of the Body. By Jonn H. Talbort, Boston, Mass.

A marked reduction of the internal temperature of the body was achieved by the technique of Smith and Fay. Thirty-five experimental periods were studied in a group of 15 persons under 40 years of age. All were suffering from severe mental disorders but were essentially normal otherwise insofar as could be determined by clinical examination of the internal organs. The experimental periods varied from 24 to 72 hours. Internal temperatures as low as $75^{\circ} \mathrm{F}$. were observed. Restoration of body temperature was uneventful and no serious complications or consequences were noted. Extensive physiological, biochemical, and clinical data were collected during each low temperature period. These concern (1) respiratory function of the blood, (2) carbohydrate metabolism and (3) cardio-vascular-renal function.

Changes in the third category are discussed in this communication. The size of the heart, as measured by the teleoroentgenogram, decreased slightly. Cardiac arrhythmias appeared when the rectal temperature fell below $85^{\circ} \mathrm{F}$. These and other variations were recorded on the electrocardiograph. A pronounced constriction of the arteries, veins and capillaries of the periphery appeared early in each experimental period and persisted. The peripheral blood pressure was frequently unobtainable for several hours. The time for blood to pass from arm to lung was increased. Evidence of reduction of circulating blood in the internal organs was obtained from the studies of renal function. Clearance of inulin, creatinine and diodrast by the kidneys, as well as determination of maximum tubular activity with high concentrations of glucose and diodrast in the blood, was investigated. All measured functions of the kidney were reduced by as much as $\mathbf{5 0}$ per cent. An approximate temperature coefficient was demonstrated, i.e., the lower the internal temperature of the body, the greater the reduction in kidney function. Following restoration of body temperature the kidney function was normal.

\section{The Comparative Effects of Liver and Kidney Disorders on the Proportions of Phosphatide Phosphorus, Free and Ester Cholesterol in Blood Serum. By Edwin F. GildEA and (by invitation) Evelyn B. MAN, New Haven, Conn.}

Previously reported investigations, indicating that serum phosphatide phosphorus varies directly with changes in total cholesterol in normal, hyper- and hypothyroid and malnourished subjects, have led to a search for disorders in which this relation might be disrupted.

In 14 of 30 patients with a number of symptoms of severe liver disease, the proportions of phosphatide phosphorus to total cholesterol deviated markedly from the normal, the phosphorus fraction being relatively high. As the liver disease progressed, both lipoid components ultimately decreased to below normal levels. These changes tended to parallel the amount of reduction in esterified cholesterol.

Similar data on 8 patients with nephrosis also revealed a disproportion between phosphatide phosphorus and cholesterol but in the opposite direction from that in the patients with liver disease. When remissions occurred in the nephrosis these lipoid components were restored to normal proportions.

In 26 patients with chronic nephritis (chiefly glomerular in type in most cases), the amounts of phosphatide phosphorus and cholesterol approximated normal proportions. Five of these patients, however, had abnormally high lipoids with comparatively low phospholipid phosphorus, as in the nephrosis cases. As the nephritis became more severe, the lipoids tended to fall to below normal levels and the lipoid components returned to normal proportions.

In addition to demonstrating the intimate association of phosphatide and cholesterol metabolism, the above findings contribute to the already extensive data indicating that the liver plays a fundamental rôle in lipoid metabolism, and suggest that the kidneys may take a more important part in controlling blood lipoids than has commonly been attributed to them.

Pathological Variations in Blood Pyruvic Acid.* By Ernest Bueding, Herman Wortis and Martin H. Stein (by invitation) and Norman Jollifre, New York, N. Y.

Cocarboxylase (thiamin pyrophosphate) is necessary for the normal catabolism of pyruvic acid. We have deter-

* Aided by grants from the John and Mary R. Markle Foundation and the Williams-Waterman Fund of the Research Corporation. 
mined blood pyruvic acid levels in 280 individuals and have obtained the following results :

1. In 39 normal adults, the fasting pyruvic acid level in the blood ranged from 0.77 to $1.23 \mathrm{mgm}$. per $100 \mathrm{cc}$., the average being 1.00 .

2. In 79 individuals with "functional" psychoses and psychoneuroses, the values ranged from 0.71 to $1.29 \mathrm{mgm}$. per 100 cc., the average being 0.98 .

3. In 35 patients with organic neurological disease without evidence of nutritional deficiency, the values ranged from 0.63 to $1.17 \mathrm{mgm}$. per $100 \mathrm{cc}$., the average being 0.97 .

4. In 56 patients with various medical disorders not complicated by fever or nutritional deficiency, the values ranged from 0.49 to $1.31 \mathrm{mgm}$. per $100 \mathrm{cc}$, , the average being 0.94 .

5. In 33 alcohol addicts who showed no evidence of acute peripheral neuropathy, the values ranged from 0.54 to $1.12 \mathrm{mgm}$. per $100 \mathrm{cc}$., the average being 0.92 .

6. In 38 cases associated with thiamin deficiency (acute peripheral neuropathy in alcoholics, Wernicke's syndrome and beriberi heart disease), the values ranged from 1.43 to $3.63 \mathrm{mgm}$. per $100 \mathrm{cc}$., averaging 1.92 .

It has also been demonstrated that, following the ingestion of glucose (1.75 $\mathrm{mgm}$. per $\mathrm{kgm}$.), there is a rise in blood pyruvic acid, and in normal subjects the curve follows a fairly characteristic pattern, generally returning to normal in three hours.

Finally, evidence is presented to show that, in cases associated with thiamin deficiency, the pyruvic acid curve following glucose ingestion is abnormally elevated, and fails to return to the fasting level. Following therapy with thiamin, the fasting pyruvic acid level and the pyruvic acid curve return to normal.

The Effects of Tyrosinase on Arterial Hypertension. By Henry A. Schroeder and Mark H. Adams (by invitation) and Alfred E. CoHN, New York, N. Y.

An attempt has been made to learn something of the nature of the pressor substance or substances believed to be responsible for arterial hypertension. According to Holtz, decarboxylation but not deamination of certain amino acids occurs in the ischemic kidney, leading to the formation of pressor amines. As the most powerful pressor amines are phenols, tyrosinase was used. This phenolic oxidase was found to lower the blood pressure of rats and dogs made hypertensive as the result of experimental renal ischemia. It was therefore injected into human beings. Twenty patients exhibiting arterial hypertension of various stages were given daily subcutaneous injections of this enzyme. In one there was little effect; in three the blood pressure fell to slightly lower levels. In the remainder it appeared that the disease temporarily was definitely altered. This was accompanied by changes in the level of blood pressure, regression of hemorrhagic and exudative lesions in the ocular fundi (eleven cases), diminution in the size of the heart in x-ray photographs (five cases), diminution in the level of the urea nitrogen in the blood without change in urea clearance (nine cases), increase in the clearance of urea (six cases) and disappearance of symptoms, such as headache (ten cases), palpitation (seven cases), dyspnea and orthopnea (two cases) and edema (one case). In nine, electrocardiograms were altered in the direction of normal.

This enzyme, a protein, acts as an antigen. In four individuals severe allergic reactions developed at the site of injection. Changes in the level of blood pressure occurred before these appeared.

When injections were stopped, the blood pressure soon (within three to seven days) returned to its previous level. Subsequent series of injections were given to three patients with similar results. Because this enzyme is specific for phenolic compounds, it is believed that a phenolic substance common to some hypertensive states is altered. It is not yet known that this phenol is the pressor substance but the fact that the pressor action of angiotonin is directly inactivated by tyrosinase suggests that hypothesis.

The Nature of the Arterial Hypertension Produced in Normal Subjects by the Administration of Angiotonin. By Robert W. Wilkins and (by invitation) Chardes N. Duncan, Boston, Mass.

Angiotonin administered intravenously in single doses or by continuous infusion to normal subjects caused increases of systolic and diastolic arterial pressure, rises of venous pressure, and decreases of vital capacity proportional to the dosage used. During the hypertension there was bradycardia, and a decrease in the stroke volume of the heart, resulting in a striking reduction of cardiac output (ballistocardiographic). Teleoroentgenogram revealed usually a small increase in heart size, never a decrease. In the limbs there was often a decrease in blood flow as measured plethysmographically or by skin temperature, but usually within the normal range. Blood flow during reactive hyperemia (after 5 minutes' arterial occlusion) in the muscular segments increased with the rise of arterial pressure. Sympathetic vasomotor reactions were retained, and the pressor response to the cold test of Hines and Brown was not altered. Circulation time often increased, but remained within normal limits. Spinal fluid pressure was not significantly changed. Mild symptoms of dizziness, substernal oppression, nausea or palpitation were occasionally noted. The effects continued at a stable level during a constant infusion, and they subsided 4 to 8 minutes after the cessation of the administration, whether infusion or single injection.

Unilateral Renal Function in Essential Hypertension. By Herbert Chasis and Jules Redish (introduced by William S. Tillett), New York, N. Y.

The clearance method has been used in unilateral studies in order to obtain evidence on the question as to whether unilateral renal ischemia is present in patients with arterial hypertension.

Twenty patients with essential hypertension have been studied. The observation started with a cystoscopic examination, after which ureteral catheters were inserted 
for a distance of $12 \mathrm{~cm}$. The cystoscope was then removed and a urethral catheter was placed in the bladder in order to detect extra-catheter leakage. Urine and blood samples were collected in the usual manner of the clearance technique. Effective renal blood flow, filtration rate and tubular excretory mass were measured in the separate kidneys. Finally, retrograde pyelography was done in the supine and sitting positions.

Diodrast clearance and $\mathrm{Tm}$ were measured in 10 patients, using both ureteral and bladder catheter techniques. The results were in agreement. The unilateral methods also gave results in normal individuals which were comparable to those obtained in a large series of normals studied by the bilateral technique.

The absolute rate of glomerular filtration was usually reduced in hypertensive subjects. This has been interpreted as indicating obliteration of glomeruli in both kidneys.

The tubular excretory mass was reduced in every hypertensive subject studied. These findings are interpreted as indicating a progressive destruction of renal tubular tissue.

If those patients in whom renal hyperemia is present as a result of either increased glomerular pressure or impotent tubules are omitted, the blood flow per unit of tubular mass was found to be subnormal in every kidney.

This renal ischemia is primarily caused by efferent arteriolar constriction, since the reduction in blood flow is associated with an increase in the filtration fraction.

The specific gravity of urine simultaneously collected from either kidney usually is similar, but occasionally the kidneys excrete different concentrations of urine at the same time, in spite of an equal blood flow, filtration rate and tubular mass. Observed inequality in urine flow is important in that excretory tests of renal function can readily be misinterpreted. Urinary dye concentrations may vary from the two kidneys and yet their blood flow and functional capacity may be equal. This point is also significant in intravenous pyelography, since differences in concentrations of diodrast referable solely to differences in urine flow may result in differences in densities of the $\mathrm{x}$-ray shadows.

Abnormal pyelographic findings are not necessarily associated with functional changes, and marked functional disparity in the two kidneys may not be associated with abnormal retrograde pyelograms. Many of the variations in uretero-pyelograms are believed to be without significance.

As the destruction of the renal parenchyma progresses in hypertensive disease, the blood flow per unit of tubular excretory mass remains equal in the two kidneys. In no instance in this study was there any indication of a unilateral ischemic kidney.

Adenomatous Hyperplasia of the Adrenal Cortex in Essential Hypertension. By James F. RiNeHART and (by invitation) O. O. Williams and William S. CappeILER, San Francisco, Calif.

Adrenal glands removed routinely at necropsy have been carefully weighed and studied. In a series of 26 cases of essential hypertension, the mean weight of the glands has been found to be approximately one-third greater than in a control group of 100 non-hypertensive cases. The enlargement is due to a nodular or adenomatous hyperplasia of the cortex. This is frequently quite striking and is found to some extent in practically all cases. Microscopically the cortical cells are arranged in irregular, tortuous columns. The cells are hyperplastic and show a high lipoid content. Changes of this type are infrequently seen in non-hypertensive cases. One of 4 cases of glomerular nephritis and 2 of 9 cases of chronic pyelonephritis showed adenomatous hyperplasia of the adrenal cortex comparable to that seen in essential hypertension and 8 of the cases of chronic pyelonephritis showed more than the average amount of lipoid in the cortical cells. This suggests that some stimulation of the adrenal cortex may result from a renal type of hypertension. While the adrenal cortical hyperplasia which we have shown to occur commonly in essential hypertension may also be a secondary effect, it is suggested that a primary hyperplasia and hypersecretion of the adrenal cortex might induce hypertension. In view of the anatomic evidence presented and other data cited, this possibility seems worthy of further investigation.

Factors Causing Increase in Venous Pressure of the Lower Extremities During Abdominal Operations. By David Davis and (by invitation) Samuel GILMaN, Boston, Mass.

The venous pressure in the lower extremities has been studied during and following abdominal and pelvic surgery. Four factors produced appreciable increases in venous pressure: (1) packing or traction of certain structures such as the uterus or gall bladder; (2) the Trendelenburg position; (3) the abdominal binder; and (4) postoperative abdominal distention.

During 7 operations on the uterus, 5 of which were for hysterectomy, increases in venous pressure above $40 \mathrm{~cm}$. of water were maintained for one-half to one and onehalf hours; in 5 operations there were transient rises above $60 \mathrm{~cm}$. and in two, such rises were maintained for 18 to 33 minutes.

These increases were partly due to the Trendelenburg position. It was found that this position alone caused increases in venous pressure proportional to the angle of the table. In one case, for example, as the head of the table was dropped 10 degrees, venous pressure increased $15.5 \mathrm{~cm}$.; at an angle of 20 degrees, $34.0 \mathrm{~cm}$.; and at an angle of 30 degrees, $49.0 \mathrm{~cm}$. of water above the horizontal level. These increases generally persisted as long as the Trendelenburg position was maintained. Studies with Dr. A. S. Freedberg showed that the femoral-tocarotid sinus circulation time in dogs is considerably slowed with the assumption of the Trendelenburg position.

In 2 of 3 operations for cholecystectomy less striking but appreciable rises in venous pressure were noted during packing or traction on certain structures. Few changes, on the other hand, were observed in the course 
of 5 operations for unilateral inguinal hernia. The application of the binder in 3 of these, however, produced an immediate and significant rise in venous pressure; in one an increase of $12 \mathrm{~cm}$. persisted during the 30 -minute period of postoperative observation. In 2 patients with postoperative distention the femoral venous pressures were elevated.

With marked and at times prolonged increases in venous pressure the possibility of injury to the venous bed and subsequent thrombophlebitis must be entertained. Furthermore, factors causing increases in venous pressure either as a result of mechanical obstruction or irritation leading to contraction of veins may continue to operate in the post-operative course. A tight abdominal binder may retard venous circulation, and this is possibly true of a loose one when abdominal distention develops.

Demonstration that the Cell Plasma Ratio of Blood Contained in Minute Vessels is Lower than That of Venous Blood. By Eugene A. Stead, Jr. and (by invitation) Richard V. Ebert, Boston, Mass.

Direct observation of the minute blood vessels has shown that the red cells flow in the rapidly moving central portion of the stream and that there is a slow moving clear layer of plasma adjacent to the wall of the vessel. Because of the size of this peripheral layer of clear plasma in the minute blood vessels, it has been suggested that the cell plasma ratio of blood contained in the minute vessels is lower than that of blood from the large vessels.

The cell plasma ratio of the blood normally contained within the minute vessels has not been previously determined because of the difficulty in obtaining a sample of this blood. Blood collected from a vein or from the cut ends of minute vessels is representative of the blood flowing from the minute vessels but it is not necessarily representative of the blood contained within the minute vessels. The blood contained within the minute vessels consists not only of the central core of red cells and plasma which is flowing rapidly into the veins, but also of the slower moving peripheral layer of plasma.

A portion of the blood normally contained within the minute vessels was obtained by the following technique: (1) the arterial inflow to the arm was obstructed; (2) the blood from the larger vessels was removed by needle and syringe; (3) the blood normally contained within the minute vessels was then forced into the veins of the forearm by applying an Esmarch's bandage.

In 15 experiments the hemoglobin concentration of the blood milked from the minute vessels was from 0.8 to 1.8 grams lower than the hemoglobin concentration of venous blood. Determinations of serum protein concentration and of hematocrit reading showed that the lower hemoglobin concentration of blood normally contained within the minute vessels could not be accounted for by the entrance of extracellular fluid into the blood stream or by a shift of fluid from red cells to plasma.

The following conclusions have been drawn:

1. The venous blood is richer in cells and poorer in plasma than the blood contained within the small vessels.
Therefore, the cell plasma ratio of blood drawn from artery, vein, or finger is not representative of the cell plasma ratio of the entire circulating blood.

2. The value for the red cell volume as calculated from the plasma volume and hematocrit reading is falsely high because of the uneven distribution of cells. The value for the total circulating hemoglobin, as calculated on the basis of the plasma volume, hematocrit reading and hemoglobin concentration is also falsely high.

3. It is not possible to quantitate accurately changes in plasma volume from the changes in hematocrit reading or hemoglobin concentration.

Pituitary-Diabetes in the Cat Treated by Low Diet, Insulin, Phlorhizin and Adrenalectomy. By F. D. W. Lukens and (by invitation) F. C. Dohan, Philadelphia, $\mathbf{P a}$.

Pituitary-diabetes in the cat provides a form of stable diabetes with a reversible island lesion (hydropic degeneration for the first 3 months) in which functional recovery of the animal and morphological restoration of the islands may be studied. Partially depancreatized cats which had recovered from the operation without diabetes were injected with crude saline anterior pituitary extract until they remained diabetic after the extract was discontinued. We have described the recovery following insulin treatment in such animals. This recovery was independent of the severity of the disease, but was limited by the duration of the diabetes and the ultimate development of irreversible lesions (atrophy and fibrosis). Results of methods other than treatment with insulin are now presented.

Reduction in diet for 24 to 26 days was followed by recovery in two cats with mild diabetes. This means that on resuming their original diet they gained weight and the blood sugar remained normal. Five cats with moderately severe diabetes were not controlled by a similar reduction in diet. Unlike insulin, the result from dietary treatment is greatly limited by the severity of the disease.

Diabetic animals recovered following the administration of phlorhizin for 2 to 3 weeks. Restoration of the islands also followed adrenalectomy. After adrenalectomy the extent of functional recovery was uncertain. The four procedures (low diet, insulin, phlorhizin, adrenalectomy) have in common a favorable influence in this type of diabetes, and all reduce the blood sugar to normal levels during treatment. As they differ in many other respects these methods should afford a new approach to the physiological analysis of this disease.

The Relation of Magnesium to the Thyroid Hormone. By Paul H. Lavietes and (by invitation) Robert F. Dine, New Haven, Conn.

The observation of Soffer and his collaborators that non-ultrafiltrable, or bound, magnesium is consistently elevated in hyperthyroidism and is absent in myxedema has been confirmed. The technique of ultrafiltration and analysis has been modified. Bound magnesium remains above normal after treatment of hyperthyroidism with 
iodine, a matter of considerable importance when diagnosis has been obscured by previous administration of iodine. In 7 patients with hypermetabolism without hyperthyroidism, bound magnesium was uniformly normal. Hyperthyroidism was excluded in these patients by observations of normal serum iodine and failure to respond to iodine therapy. Except for 5 cases of frank myxedema, in which bound magnesium was entirely absent, bound magnesium was observed to be below the lowest normal value, 11.3 per cent, in only 4 instances. Collateral clinical and laboratory evidence is adduced to indicate that these cases may actually represent partial hypothyroidism.

Data relating bound magnesium to iodine in serum suggest that magnesium may be an integral part of the circulating thyroid hormone, or of the complex in which the hormone functions.

Protein-Bound Iodine in Blood Plasma. By Willin M T. Salter and (by invitation) A. Merton BAssetr and Albert H. Coons, Boston, Mass.

Although hormone in the thyroid gland occurs as thyroglobulin, the protein-bound iodine in the blood plasma of man and of the horse was found to reside prominently in the traditional albumin fraction. The protein-bound iodine is subject to fluctuations, depending upon thyroid activity. Such fluctuations are due chiefly to the thyroxine-like moiety thereof. Although the fraction resembling diiodotyrosine may vary proportionately, because of its small magnitude it contributes very little to the absolute increment. Despite variations in the proteinbound iodine, the inorganic iodine concentration is rather low and approximately constant. These findings suggest that the protein-bound moiety of plasma iodine may be used as an objective index of circulating thyroid hormone and, indirectly, as a measure of thyroid activity.

A series of 94 cases was analyzed from this standpoint. In about two-thirds of them the clinical diagnosis and the basal metabolic rate were consistent and there was a high correlation between the latter and the protein-bound iodine. Of the remaining one-third, the basal metabolic rate did not clearly reflect the clinical status, whereas the protein-bound iodine was more reliable. In hypothyroidism, plasma protein-bound iodine was consistently low and the thyroxine-like fraction thereof almost nil. Of special interest is the exophthalmic ophthalmoplegia group, classified as "Graves' disease without hyperthyroidism," in which the basal metabolic rate was often within normal limits and the plasma protein-bound iodine was also normal.

Collection of Iodine in the Thyroid as a Differential Criterion in the Diagnosis of Two Types of Graves' Disease. By S. Hertz and A. Roberts (introduced by J. H. Means), Boston, Mass.

In another place a rather extended description of a special variety of Graves' disease in which the eye symptomatology is dissociated from the thyrotoxic element is described by Hertz, Means and Williams. We wish to present here data bearing on the difference in iodine metabolism in the two types of Graves' disease, as determined by the use of radioactive iodine as a tracer in the study of thyroid physiology. The pattern of collection of the iodine in the thyroid in ordinary Graves' disease follows a definite curve; the collection in the thyroid of the special variety has a differently shaped curve; and both of these are quite separable from the curve for normal patients. In general, the method used was as follows: one milligram of labelled iodine was administered by mouth, and the iodine uptake in the gland was measured at various time intervals by means of a Geiger-Müller counter externally placed over the thyroid.

\section{The Effect of Aluminum Hydroxide Ingestion on the} Phosphorus and Calcium Disorders of Hypoparathyroidism. By Fuller Albright and (by invitation) Charles H. Burnett, William Parson, and Hirsch W. SUlkowitch, Boston, Mass.

Aluminum hydroxide has long been used in the production of experimental rickets in animals. It produces its effect by uniting with phosphates in the gastro-intestinal tract and preventing their absorption. Inasmuch as it has for several years been the opinion of those in this laboratory that the disorder of calcium metabolism in hypoparathyroidism is dependent on a more fundamental disorder in phosphate metabolism, it seemed of interest first to determine whether the administration of aluminum hydroxide would lower the high serum phosphorus level in hypoparathyroidism by preventing phosphate absorption and, secondly, whether it would elevate the low serum calcium value. Such was found to be the case, although the results were not quite those that would have been predicted. The studies include complete metabolic data on one patient.

Replacement of Potassium by Sodium in Muscles of Normal Dogs Receiving Desoxycorticosterone Acetate. By Joseph W. Ferrebee, Donald Parker, Wimlam H. Carnes, and Mildred K. Gerity (by invitation) and Dana W. Atchley and Robert F. Loeb, New York, N. Y.

Normal dogs receiving daily subcutaneous injections of 25 milligrams of desoxycorticosterone acetate develop diabetes insipidus and attacks of profound muscular weakness. In experiments on six normal animals it was found that administration of desoxycorticosterone acetate caused an increase in intracellular sodium and a decrease in intracellular potassium of skeletal muscle, but no change in the extracellular water jacket of the muscle, that is, no change in the so-called chloride space. The changes in intracellular sodium and potassium concentrations could be prevented by the administration of potassium chloride which maintained a normal relationship of sodium to potassium in the cell and prevented the occurrence of paralysis. The diabetes insipidus was independent of the muscle electrolyte pattern and developed whether or not the animals were given potassium chloride. The diabetes insipidus could be correlated with the fact that all the animals receiving hormone had an elevation of serum 
sodium concentration. On the other hand, a high serum sodium concentration was not responsible for the increase in muscle sodium. The serum sodium was elevated in the animals on potassium chloride in whom no disturbance of muscle electrolyte concentrations was observed. This latter circumstance offered evidence that sodium goes into muscle cells only when potassium comes out, that is, sodium will replace but not displace potassium. This view is consistent with the idea that desoxycorticosterone, by increasing the renal excretion of potassium, lowers the serum potassium concentration and shifts the equilibrium of potassium between serum and cell in the direction of an increased loss of potassium from the cell.

\section{The Peripheral Nature of the Muscular Weakness of} Familial Periodic Paralysis. By George D. Gammon and A. M. Harvey, Philadelphia, Pa.

Myograms made during the development of the attack show a gradually rising threshold to electrical stimulation of the motor point, failure of response to single shocks, then to stimuli below 15 per second, and finally complete failure to any frequency of stimulation.

Electromyograms from the short flexor of the fifth finger are of low voltage and of nearly monophasic form in belly-tendon leads, but leads more closely spaced show increasingly diphasic responses. This suggests that the impulse fails to spread throughout the length of the muscle fiber.

Circulatory obstruction of the arm during an attack lowers the threshold and increases tension and voluntary strength. The voltage of the electromyogram increases, and its form becomes more diphasic.

The potassium salts restore strength and reverse the changes described above. At the onset of recovery a tetanus clearly augments the responses, just as exercise will hasten recovery.

In the attack the disturbance involves the muscle fiber and the action potential appears not to be conducted throughout its length. The defect is restored by maneuvers which probably increase the potassium outside the muscle fiber, relative to that inside, by a loss of fiber potassium during exercise or asphyxia or by an increase of serum potassium from administration of the salt.

Studies in Muscular Tension in the Neuroses. By JURGEN RUesch (by invitation) and JACOB E. Finesinger, Boston, Mass.

Muscular tension was studied in a series of 38 psychoneurotic patients of varied diagnoses and 12 normal control subjects by the use of two methods. The first method made use of a stylus, the point of which was attached to a rubber bulb to record the pressure exerted on the point (point pressure) during writing. The stylus was enclosed in another rubber bulb which was held by the subject during writing to obtain the grip pressure. Each bulb was connected by means of pressure tubing to a Marie tambour. The oscillations of the tambours were photographed on a moving film and in this way. records of the point and grip pressure used during the writing of a standard paragraph could be calibrated and studied. After the records of handwriting and pressure had been made, the subjects were given a questionaire to determine their mental status with special reference to feelings of general and neuromuscular tension. The second method involved the study of electromyographic tracings obtained from the extensor and flexor forearm muscles of the same group of patients and control subjects while they were asked to make a fist at given time intervals. From the electromyographic tracings the time taken for the motor activity and for relaxation could be measured.

The results obtained indicated the following:

1. The writing time for the patients was considerably greater than that of the control group.

2. The patients showed a greater variation in both point and grip pressure than did the control subjects.

3. Greater values for grip pressure were obtained in the group of patients than in the control group.

4. The group of patients had a much shorter relaxation time between discrete muscular activity (making a fist) than did the group of normal controls.

5. There was a strikingly positive correlation between high values for grip pressure and the presence of neuromuscular tension as reported by the subjects.

Involuntary Activity in Skeletal Muscle in Joint Disease: Electromyographic Observations. By CHARLES L. Short and (by invitation) Alfred O. Ludwig and Robert S. Schwab, Boston, Mass.

Muscle atrophy is one of the most disabling features of rheumatoid arthritis. The factors responsible have not yet been established, although clinical and experimental evidence suggests a reflex mechanism from impulses set up in the diseased joints.

We have recorded electromyograms from patients with joint disease by means of a Loomis ink-writing oscillograph. While the tracings of voluntary contractions demonstrated no constant deviation from the normal, many of the patients showed action potentials while immobile and apparently relaxed. These usually consisted of a series of regular diphasic spikes at a rate varying from 6 to 15 per second and with an amplitude as high as 40 microvolts. Such patterns were found in 10 of 20 patients with rheumatoid arthritis, as well as in gonorrheal arthritis and joint limitation due to fixation. The activity usually appeared and disappeared spontaneously, might alternate from spot to spot within the muscle, and might be present in one muscle related to a joint and not in another at the same time. In certain cases, the series of spikes could be made to disappear on altering the position of the limb, but in other cases, it persisted, uninfluenced by voluntary and reflex contraction of the muscle.

The nature and origin of this involuntary muscle activity cannot be defined at present, but consideration is given to the possibilities that 
1. It arises by a reflex path from impulses set up in the joint.

2. It is a factor in the production of muscle atrophy in joint disease.

\section{READ BY TITLE}

The Host Factor in Protection by Vaccine Immune Serum. By R. F. PARKer and (by invitation) R. H. GreEn, Cleveland, O.

Early experiments gave results which were interpreted as indicating that infection with the virus of vaccinia might follow the suitable introduction of a single viral particle. Later work indicated, however, that while this was true for fully virulent strains, it did not hold when strains of lower virulence were tested, the evidence seeming to indicate that under these conditions it was the chance of entry of the virus into a susceptible cell which determined whether or not infection occurred. In respect to the proportion of cells susceptible, variation between animals was observed. In the protection test with immune serum it has been found that, with increasing amounts of serum, the regularity of the titration curve decreased. The present experiments indicate that this is due primarily to variation between the response of individual animals. It is suggested that the action of immune serum is to render a certain proportion of the host cells refractory to infection, and that animals differ in the proportion of cells so protected by a uniform concentration of serum.

The Weil-Felix Reaction in Proteus and Pyocyaneus Infections. By F. Tremaine Billings, Jr. and Gustave J. Dammin (introduced by Clifford L. Derick), Boston, Mass.

The present study was suggested by the observation that the sera of many patients with Proteus and Pyocyaneus infections possessed high agglutinin titers for the Weil-Felix antigens. The incidence of positive Weil-Felix reactions was found to be higher in patients with these infections than in a large series of normal controls.

In a group of 9 patients, 8 with Proteus infections and 1 with a Pyocyaneus infection, agglutinin tests were carried out with Proteus $\mathrm{OX}_{10}, \mathrm{OX}_{2}, \mathrm{OXK}$ and the infecting organism. Of this group, 5 possessed agglutinins for Proteus $\mathrm{OX}_{19}$ in a serum dilution of 1:40 or higher, and also for the infecting organism in high titer; the remaining 4 failed to show agglutinins for either $O X_{19}$ or the infecting organism. Five patients agglutinated Proteus $\mathrm{OX}_{2}$ in serum dilutions ranging from $1: 40$ to $1: 5000$, and 8 agglutinated Proteus OXK in serum dilutions of $1: 80$ to $1: 2560$. The sera of these 9 patients were absorbed with the infecting organism and in each instance it was possible to remove partially or completely the Weil-Felix agglutinins, indicating the presence of a common antigen.

Rabbits immunized with these 9 strains developed strong Weil-Felix reactions. Six agglutinated Proteus OXK in dilutions of $1: 2560$ or higher; 7 agglutinated Proteus $\mathrm{OX}_{2}$ in serum dilutions ranging from 1:40 to $1: 10000$, and one rabbit developed a high agglutinin titer for Proteus $\mathrm{OX}_{12}$.
In each instance it was possible, with the immunizing organism, to absorb out the Weil-Felix agglutinins.

It is concluded that a positive Weil-Felix reaction may develop during the course of Proteus or Pyocyaneus infections. In view of the general use of the Weil-Felix reaction in the diagnosis of rickettsial diseases, the results here reported suggest the need of greater care in interpreting this non-specific test.

Solubility Studies on the Oral Administration of Sodium Sulfapyridine. By SIDNEY S. SoBIN (introduced by Arthur C. Curtis), Ann Arbor, Mich.

Oral therapy with sodium sulfapyridine has been recommended by Ratish, Davidson, and Bullowa (J. Pharm. and Exper. Therap. 1940, 69, 365) as a procedure for rapidly achieving high blood levels. This procedure must assume an alkaline gastro-intestinal medium to maintain the sodium salts in solution. We have investigated the neutralizing effects of gastric juice on sodium sulfapyridine and the clinical absorption of these drugs.

Electrometric titration and quantitative sulfonamide determination of a $\mathbf{5}$ per cent solution of sodium sulfapyridine with gastric juice demonstrated that precipitation of the free base occurs with small amounts of gastric juice and is complete when the $\mathrm{pH}$ of the solution drops below 8.5 to 9.0 .

Equivalent amounts of sulfapyridine and sodium sulfapyridine in aqueous solution or acacia suspension were administered to a group of patients. Blood levels of free drug were similar with both compounds. Some patients showed a more rapid rise but no higher peak (maximum level) with the sodium salt. Administration of equal amounts of sulfapyridine in tablet form and in finely powdered suspension again showed similar curves but more rapid absorption with the suspension. This suggests that particulate size may be an important factor in absorption.

Two patients showed an unexpected rapid high absorption curve with sodium sulfapyridine. Neither had free fasting $\mathrm{HCl}$. Repetition of the sodium sulfapyridine administration, after histamine had produced acidity, showed a response similar to that of the free base. The $\mathrm{pH}$ of the gastric juice in this and other cases showed only slight elevation with oral sodium sulfapyridine.

\section{Discrepancies in the Inhibition-Concentration Relationship} of the Sulfonamides. By JEROME S. HARrIS and HENRY I. КоH (introduced by David T. Smith), Durham, N. C.

In the case of certain sulfonamide drugs, e.g., sulfapyridine in pneumonia, it is becoming apparent that the therapeutic effect is independent of the dose or blood concentration over a fairly wide range. Although this paradox may be due to factors in the host or host-parasite relationship, it may be explained, in part, by the response of the bacteria to changes in drug concentration.

This may be demonstrated by comparing the inhibitions produced by varying concentrations of the sulfonamides on the growth rate of $\mathrm{E}$. coli in proteose peptone medium. 
Thus, raising the concentration of sulfapyridine from 2 to $60 \mathrm{mgm}$. per cent merely increased the inhibition of growth from 74 to 80 per cent. Above and below these levels, the inhibition increased rapidly with increase in drug concentration. Similar results were obtained with sulfathiazole and sulfadiazine. However, raising the concentration of sulfanilamide from 2 to $20 \mathrm{mgm}$. per cent increased the inhibition from 13 to 68 per cent. This suggests that raising the concentration of the sulfonamides, sulfanilamide excepted, may not produce the desired increase in therapeutic effect.

To explain these results, we suggest that the sulfonamides affect directly or indirectly a number of different bacterial metabolic systems at different concentrations. One such system, affected at low drug concentrations, is the synthesis of methionine in E. coli.

Studies on Absorption of Sulfapyridine and Sulfathiazole. By J. Murray Kinsman and (by invitation) ЈоHN Walker MoOre, Louisville, Ky.

In 1939 we studied the absorption of sulfapyridine following the oral administration and following the rectal administration of its sodium salt. These studies were reported in part two years ago. In 1940, and to date in 1941, we have been studying the absorption of sulfathiazole. This has been administered orally and its sodium salt has been given rectally, intramuscularly and (lately) orally.

This report deals with a comparison of the absorption of the drugs under the above conditions.

Sulfapyridine is absorbed and excreted more slowly than sulfathiazole and maintains a higher blood concentration for equivalent dosage. Because of the rapidity of excretion of sulfathiazole, to be effective, doses have to be given at more frequent intervals than for sulfapyridine. By rectum, very high blood concentrations can be obtained and maintained with sodium sulfapyridine but not with sodium sulfathiazole. Intramuscularly, sodium sulfathiazole is slowly absorbed and gives blood concentrations much below what one would expect from corresponding oral doses of sulfathiazole; moreover, over a period of several days less than 25 per cent of the amount given can be recovered in the urine, indicating that some sort of protective coating may develop about the deposit in the muscle, preventing its ready absorption. The work on sodium sulfathiazole by mouth is in progress now, but so far it seems to indicate that the blood concentrations are lower than from the sulfathiazole itself.

Sulfathiazole was given to one patient following a total gastrectomy, and blood concentrations were below the expected level.

Protective Activity of Normal Human and Animal Sera for Sulfapyridine-Treated Mice Infected with Pneumococci. By Sophie Spicer (by invitation) and Wheelan D. Sutliff, New York, N. Y.

Using mice fed a diet containing 1 per cent sulfapyridine as test animals, blood sera from other mice, guinea pigs, rabbits, rats, dogs, infant humans and adult humans were examined to find the relative activity of these sera in protecting against pneumococcus infection, Type I.

The sera of dogs and adult humans increased the survival rate of sulfapyridine-treated mice. The sera of mice, guinea pigs, rabbits, rats and human infants did not increase the survival rate of sulfapyridine-treated mice.

Further studies of fresh serum as compared with heated or "old" serum showed that the protection partially disappeared on heating or on standing. The residual activity in "old" serum was destroyed by absorption with homologous type-specific pneumococcus cultures.

Variations in Complement Activity of Blood Serum in Pneumonia. By David D. Rutstein and William H. WALKER (introduced by L. Whittington Gorham), Albany, N. Y.

The complement activity of 34 specimens of blood serum from 25 normal individuals and all but one of 47 specimens from 47 pneumonia patients following recovery fell within the narrow range of 0.00226 to $0.00660 \mathrm{ml}$.

The blood serum of 10 of 62 patients ( 16 per cent) showed, at the time of admission to the hospital during pneumonia, a marked drop ranging from $0.00773 \mathrm{ml}$. to complete failure of hemolysis with the largest amount of serum tested, i.e., $0.2 \mathrm{ml}$. of undiluted serum.

The serums from 7 of 12 pneumonia patients observed immediately before and after the administration of the initial therapeutic dose of antipneumococcic serum showed a striking drop, following serum administration, to a similar low range of complement activity.

The serums from all of 12 pneumonia patients observed immediately before and after the administration of the initial therapeutic dose of sodium sulfathiazole or sodium sulfadiazine showed no significant changes in complement activity following serum administration.

During serum sickness, following recovery from pneumonia, serums from 6 of 11 patients showed similar sharp decreases in complement activity which returned to normal following recovery from serum disease.

Except in one instance, the complement activity of guinea-pig serum was not inhibited by these serums of low titer after heat inactivation.

Studies on Leptospiral Infections. By Thomas G. WARD (by invitation) and Thomas B. TURner, Baltimore, Md.

Since September of last year Leptospira icterohemorrhagiae has been recovered from three cases of Weil's disease in Baltimore. Two of these patients, brothers, aged nine and eleven, were infected by drinking water from a well in which leptospira virulent for guinea pigs were repeatedly demonstrated. Another case was traced to the same source. The third patient from whom the organism was recovered worked as a chicken picker in an establishment where rats were abundant. Virulent leptospira were demonstrated in the rats.

Agglutination and complement fixation tests have been made on sera from over 400 persons belonging to various groups in Baltimore with the following result: 


\begin{tabular}{|c|c|}
\hline Number tested & $\begin{array}{l}\text { Per cent } \\
\text { positive }\end{array}$ \\
\hline Chicken pickers $\ldots \ldots \ldots \ldots \ldots \ldots 74$ & 18.9 \\
\hline Meat packers $\ldots \ldots \ldots \ldots \ldots \ldots$ & 6.2 \\
\hline Candy makers $\ldots \ldots \ldots \ldots \ldots \ldots$ & 0 \\
\hline Medical students $\ldots \ldots \ldots \ldots \ldots \ldots 66$ & 0 \\
\hline Hospital patients ........... 164 & 4.9 \\
\hline Miscellaneous .............. 29 & 20.1 \\
\hline Total $\ldots . . .$. & 7.7 \\
\hline
\end{tabular}

The complement fixation is positive in only about onehalf the sera showing positive agglutination. Rarely are complement fixing antibodies demonstrable in the absence of agglutinins.

Previous studies have shown that approximately onethird of the wild rats in Baltimore harbor $L$. icterohemorrhagiae. The foregoing data indicate that, in occupations entailing immersion of the hands in water open to contamination by rats, the risk of leptospiral infection is considerable.

Five strains of $L$. icterohemorrhagiae isolated locally from three human cases, a rat, and well water, respectively, show cross agglutination and complement fixation in high titer, and differ markedly in this respect from a strain of $L$. canicola isolated from a dog.

The following conclusions may be drawn:

1. Weil's disease is more common in Baltimore than previous records indicate.

2. The risk of infection is greatest among persons whose work brings them into contact with water contaminated by rat urine.

3. The agglutination reaction is commonly positive in higher titer, and over a longer period of time than is the complement fixation test.

Sensory Neuron Degeneration in Young Pigs. Protection by Various Liver Extracts and Certain Vitamins. Antianemic Effectiveness of Livers of Ataxic and Nonataxic Pigs. By M. M. Wintrobe and (by invitation) Cecil Mushatt, Joseph L. Miller, Jr., Lawrence C. Kolb, and H. J. Stein, Baltimore, Md.

The observed efficacy of whole liver in protecting pigs against degenerative changes in the nervous system led to the investigation of the value of the various fractions of whole liver obtained during the manufacture of parenteral liver extract. The most effective fraction was an alcoholsoluble filtrate obtained after passage through permutit (parenteral anti-pernicious anemia liver extract, Parke Davis); the remaining fractions were relatively less effective in the following order: the residue after acid and heat treatment of whole liver ("press cake"), the fraction insoluble in 70 per cent alcohol ("Whipple" fraction) and the permutit absorbate. None of the fractions gave complete protection in all pigs in the doses used.

Preliminary observations suggest that certain of the newly synthesized " $B_{2}$ complex" vitamins afford protection. The content of these vitamins in the various liver fractions has been assayed.
In assays which are not yet complete the livers of ataxic pigs seem to be less effective in the treatment of pernicious anemia than those of normal pigs.

The relationship of these observations to pernicious anemia is discussed.

The Chemical Determination of Nicotinic Acid Deficiency. By Henry Field, Jr., Daniel Melnick, W. D. Robinson, and C. F. Wil.kinson, Jr., Ann Arbor, Mich.

The application of the cyanogen bromide reaction which we have used yields values for blood nicotinic acid higher than those reported by other chemical methods and in good agreement with determinations by different biological methods. Confirming most of the reports of determinations by biological methods, we have not found any significant difference in the nicotinic acid contents of the bloods of normal and deficient subjects. Using various types of dosing for " saturation tests," we have not found that nicotinic acid is removed from the blood of pellagrins faster than from that of normal subjects.

The determination of nicotinamide in urine is complicated by the presence of other pyridine compounds which react similarly. Trigonelline, the methylated form in which the greater part of nicotinic acid is excreted, occurs preformed, without antipellagra activity, and is widely distributed in vegetable materials, predominantly coffee. Depending upon the individual, a variable fraction of nicotine is excreted which reacts like nicotinic acid. A larger fraction of nicotine excreted behaves on hydrolysis like trigonelline. The hydrolytic behavior of trigonelline permits its differentiation from other pyridine compounds and at least semi-quantitative analysis.

Twenty-four-hour urine specimens collected during abstinence from coffee, tobacco, tea and chocolate have shown a secretion of nicotinic acid averaging distinctly less in deficient than in normal subjects, but not consistently less.

The difference in the urinary excretion of trigonelline in normal and deficient subjects was much greater and much more consistent. It appears that, with a standardized basal regime and a more quantitative recovery, the urinary excretion of trigonelline may be the best chemical indication of nicotinamide deficiency.

The Effect of Unilateral Paralysis of the Diaphragm on the Respiratory Mechanism. By GeORGE W. Wright and WARRINer WOODRUFF (introduced by William S. McCann), Trudeau, N. Y.

The effect of paralyzing one-half of the diaphragm on the rôle played by each lung in regard to minute ventilation, oxygen consumption, and carbon dioxide output has been studied previously in animals. Unsatisfactory technique, differences in the anatomy of the mediastinum and possible differences in the relative rôle played by the diaphragm in normal respiration have strongly militated against applying these observations to the human subject. Using the technique of bronchospirography as described by Jacobaeus and modified by Gebauer, we have studied the minute ventilation, oxygen consumption and carbon 
dioxide output of each lung simultaneously with the patient at rest before and after paralysis of one-half of the diaphragm. In each of the five cases there was a reduction (12 to 21 per cent) in the minute ventilation, oxygen consumption and carbon dioxide output on the paralyzed side. No signifigant change was found in the arterial hemoglobin saturation. The vital capacity tended to be slightly reduced, although the maximum minute ventilation showed no reduction. The resting respiratory rate was increased moderately.

Effect on the Lung Volume of Postural Changes in $\mathrm{Pa}$ tients with Orthopnea Due to Congestive Failure. By M. D. Altschule and (by invitation) N. Z ZMCHeck and A. Iglauer, Boston, Mass.

A number of authors have attempted to explain the orthopnea of cardiac decompensation on the basis of increased congestion of the lungs when the patient assumes the recumbent position. Variations in the degree of pulmonary congestion should cause changes in the volume of the pulmonary air. Accordingly, the various subdivisions of the total lung volume were measured by the method of Christie and Meakins in various positions in patients with congestive failure and orthopnea. Only patients with mild or moderate orthopnea could be studied, since severe orthopnea made it impossible to secure satisfactory measurements.

In the patients studied the volume of the residual air was not changed in recumbency. It is clear that increased dyspnea in the recumbent position was not due to an increase in the degree of pulmonary congestion. Similarly, the partial relief of dyspnea obtained by assuming the upright position was not due to lessened pulmonary congestion. Other pulmonary factors are, however, important in the genesis of the orthopnea of congestive failure. Decreases in reserve air occur regularly when the recumbent position is assumed. This decrease in reserve air has been reproduced in the upright position by applying a tight abdominal binder and therefore appears to be the result of elevation of the diaphragm by the abdominal viscera. This elevation of the diaphragm impedes respiratory action and also makes the intrapleural pressure more positive, thereby further increasing the work that must be done to maintain a given level of respiratory activity. It is not to be inferred that orthopnea is due solely to these mechanisms; additional studies have stressed the rôle of intracerebral factors in its genesis.

Mechanisms of Respiratory Failure under Barbiturate Anesthesia (Evipal, Pentothal). By HENRY K. Beecher and (by invitation) CARL A. MoYer,* Boston, Mass.

Although the variability of effect of the barbiturates is well known, its causes are obscure. Since respiratory failure plays an important part in death under these agents, the respiration has been used as a means of investigating, in dogs, several factors responsible for the

* Fellow of the National Research Council. variability of barbiturate effect. Evipal and pentothal have been studied. Since the barbiturates are qualitatively very similar in their actions, it is probable that the mechanisms described here apply to many members of the group. The respiratory response to the barbiturates when the blood oxygen is below normal, and the effect of low oxygen in temporarily masking serious overdosage, have been observed and considered in detail. The effect of a high oxygen content of the blood in depressing respiration under the barbiturates has been confirmed. The serious possibilities inherent in this action are now evident when considered together with the loss of sensitivity of the respiratory center to carbon dioxide under the barbiturates. This loss of sensitivity of the respiratory center results in a dangerous piling up of carbon dioxide in the blood. An increased sensitivity to small doses of barbiturates was observed when the blood carbon dioxide level was elevated. Finally, it was found that reflex respiratory failure could be produced by a slight increase in pressure in the airway when the subjects were deeply anesthetized and breathing one hundred per cent oxygen. This study provides information which helps to explain obscure accidents, particularly "sudden death without warning," under these agents and furnishes surer guides than have been available for the prevention of accidents in the future.

Pathogenetic Mechanisms in Hemolytic Anemia. By William Dameshek and (by invitation) EDWARD $B$. Miller, Boston, Mass.

Hemolytic anemia develops when certain blood-destroying factors overbalance those concerned with blood formation. Previous studies indicated (a) that hemolysins are important in both clinical and experimental hemolytic anemias and $(b)$ that spherocytosis and increased hypotonic fragility are probably the result of hemolysin activity upon red cells outside the bone marrow.

"Hypersplenism" may explain some cases in which splenectomy results in cure and in complete disappearance of spherocytes. The stasis-agglutination theory of Ham and Castle fails to explain the presence of easily demonstrable hemolysins in various cases and the absence of hemolytic anemia in conditions of marked stasis, i.e., polycythemia vera and splenic vein thrombosis.

Our recent experiments demonstrate that the normal erythrocyte may be damaged by (a) "simple" hemolysins which act directly (b) by " complex" hemolysins, including first "sensitization" of the red cell, then hemolysis due to complement activity and (c) by agglutinins. Red cells sensitized either by complex hemolysins or agglutinins are very fragile to mechanical means and thus liable to ready hemolysis in the circulation. Incompletely hemolyzed red cells-spherocytes-possess a diminished resistance to stasis. The factor of stasis appears to be less important than that of mechanical fragility or complement activity.

Hemolytic syndromes are due to a variety of agents- 
hemolysins, agglutinins, hereditary factors-which injure the red cell and make it vulnerable to erythrostasis, mechanical trauma in the circulation, complement activity, etc. In testing red cells in hemolytic anemia, the factors of mechanical fragility, reaction to abnormal $\mathrm{pH}$, etc. may be as important as that of an abnormal hypotonic fragility.

Hemolytic Action of Certain Organic Oxidants Derived from Sulfanilamide, Phenylhydrazine and Hydroquinone. By Charles P. Emerson (by invitation), Thomas Hale Ham and William B. Castle, Boston, Mass.

In order to explain the mechanism of the increased osmotic fragility of red blood cells, hemoglobinemia and hemoglobinuria observed in the acute hemolytic anemia of certain patients receiving sulfanilamide, the effect of possible metabolic derivatives of sulfanilamide, including $p$-aminophenol and phenylhydroxylamine, was investigated in vitro and in cats and compared to the effect of phenylhydrazine, hydroquinone and some related compounds.

Human or cat erythrocytes were incubated for from 4 to 24 hours at $37^{\circ}$ in serum or in buffered salt solution $(\mathrm{pH} 7.4)$ in the presence of oxygen with and without each drug in a concentration of 4.5 millimoles. Those compounds, or their derivatives, which may form oxidants in oxidation-reduction systems, namely, hydroquinone, $p$-aminophenol, hydroxylamine, phenylhydroxylamine and phenylhydrazine, caused the formation of methemoglobin, an increase in volume and osmotic fragility of the red cells eventually resulting in hemolysis. The effects of $p$-aminophenol and hydroquinone on osmotic fragility were completely inhibited by anaerobic conditions and that of hydroquinone was decreased in proportion to the tension of carbon dioxide. Certain organic compounds of related structure, which presumably do not act as oxidants under the conditions employed, failed to produce increased fragility of the erythrocytes when used in equimolar concentrations. These included sulfanilamide, sulfanilic acid, $p$ nitroaniline, $p$-aminobenzoic acid and phenol.

Each of the above compounds causing changes in vitro, when injected intraperitoneally into cats in doses of from 6 to $50 \mathrm{mgm}$. daily, produced changes in the peripheral blood which paralleled those found in certain cases of acute hemolytic anemia due to sulfanilamide, including the formation of methemoglobin, rapid development of anemia with hemoglobinemia and so great an increase in fragility that erythrocytes were hemolyzed in vitro in salt solutions approaching isotonicity. Sulfanilamide and $p$-aminobenzoic acid, when injected in similar doses, produced only moderate anemia and no significant change in erythrocyte fragility.

It is inferred that the hemolytic activity of compounds such as sulfanilamide, hydroquinone, $p$-aminophenol, phenylhydroxyamine and phenylhydrazine is due to the formation in vivo of certain oxidative derivatives which in vitro were demonstrated to cause increased erythrocyte fragility.
Leukopenia. The Rate of Disappearance of White Blood Cells from the Peripheral Blood in Leukopenic States. By JoHn S. LAwrence and (by invitation) Donald M. ERvin and Raymond M. Wetrich, Rochester, N. Y.

Cats which have been leukopenic due either to infectious feline agranulocytosis or irradiation of the bone marrow have been connected by means of an end-to-end anastomosis of the carotid arteries with normal animals. When thorough mixing of the blood has occurred, the animals have been disconnected and allowed to go on their own circulation. Following this, the normal animals have promptly developed a marked leukocytosis with a distinct "shift to the left," whereas the leukopenic animals have shown a rapid diminution in the total number of white blood cells in the vascular channels until their original leukopenic levels have been reached. The white blood cells disappear at the rate of approximately 1000 cells per cu. mm. per hour. Examination of sections from various tissues of these animals has failed to show any accumulation of white blood cells that would explain their disappearance from the blood.

As a working hypothesis, we have suggested that white cells normally stay in the blood only a short time, the blood serving merely as a means of transporting them to the various tissues of the body. Whether there is such a rapid disappearance of cells from the blood channels of normal animals cannot be said, but it is certainly true that in leukopenic animals of the types we have used there is a rapid disappearance of white blood cells from the vascular channels.

The Management of Hemophilia with Lyophiled Human Plasma Intravenously. By JoHN B. JoHnson (introduced by Samuel H. Bassett), Rochester, N. Y.

Five hemophilic patients have been studied. Evidence has been obtained to show that lyophiled plasma causes a similar reduction in the coagulation time to that caused by the transfusion of whole blood. The potency of the dried plasma has been shown to be greatest when it is prepared immediately after the blood is drawn from the donor. The sources of plasma have been patients with polycythemia vera or with acute left heart failure, and friends of hemophilic patients. No typing is necessary.

Using dried plasma only, tooth extractions have been made in two patients. Massive hematuria in one patient was practically cleared 48 hours after two doses of plasma, and 12 hours later the guaiac was continuously negative.

One of our patients had led a wheel chair existence for 3 years because of frequent recurrence of hemarthroses when walking. With weekly injections of plasma and proper muscular exercises, he began walking. For two months on this treatment no hemorrhages occurred, and the patient secured a steady job. Plasma was then discontinued for one month, during which time one traumatic and two spontaneous hemorrhages occurred (one a massive hematuria).

Dried plasma offers a simplified method for managing the emergencies of hemophilia and also a relatively in- 
expensive replacement therapy by which the hemophilic patient may be rehabilitated into the community.

The Detection of Abnormal Erythrocytes by Opacity Measurements of Blood in Varying Concentrations of Saline. By Benjamin Alexander (introduced by Samuel L. Gargill), Boston, Mass.

The opacity of saline solutions of normal red cells and cells in pathologic conditions has been measured with the Evelyn photoelectric colorimeter.

When a given number of normal red cells is suspended in 2.5 per cent saline solution the opacity is $83 \pm 3$ per cent greater than when the same number of red cells is suspended in physiological saline. In normal blood, therefore, the increase in opacity in the 2.5 per cent saline suspension is proportional to the number of red cells suspended. In certain pathological conditions, on the other hand, the red cell opacity in the two media did not show the same change as in normal blood suspension of the same number of red cells. It was found that 84 per cent of those cases showing a red cell opacity change greater than normal in suspensions of a given number of red cells had clinical conditions known to give rise to macrocytic anemia. In many instances, abnormally large opacity change was associated with a demonstrable increase in the mean corpuscular size. In some instances in which the opacity change was abnormally great, however, no abnormality of the blood could be demonstrated. In certain of these cases the opacity change became normal after liver therapy.

It would appear that this measurement of the opacity difference of a given number of red cells in physiological saline and in 2.5 per cent saline solution can be utilized to reveal a fundamental abnormality of the red cells. Thus far, most of the cases in which this abnormality has been detected have had clinical conditions known to be associated with macrocytosis.

\section{An Analysis and Correlation of Hemocytologic with Other}

Significant Reactions in Monkeys Following Simultaneous or Successive Inoculations with the Virus of Epidemic Influenza and Hemolytic Streptococci. By Charles A. Doan and (by invitation) Oram C. Woolpert, Cesar Merino, J. L. Schwab, and S. Saslaw, Columbus, $\mathrm{O}$.

The virus of epidemic influenza (PR8 strain of Type A) and the streptococcus hemolyticus (Group C) were administered either simultaneously or successively, by the nasal route, to monkeys (Macacus mulatta) under ether anesthesia. The clinical, hemocytologic, serologic, and other pertinent reactions were followed until recovery (or rarely death) occurred. The order of administration of the two agents was reversed in separate series of animals and the interval between inoculations was varied. In selected instances, recovered animals which had received one or both infecting agents were later reinoculated and comparative observations were recorded.

Primary inoculation with the streptococcus was characterized by an immediate polymorphonuclear leukocy- tosis, with little or no change in the opsonocytophagocytic index. Primary inoculation with the influenza virus, on the other hand, was followed promptly by a granulopenic leukopenia, with specific virus-neutralizing antibodies demonstrable a little later. When the virus was given concurrently with the streptococcus, or shortly thereafter, the streptococcus-stimulated granulocytosis dedeveloped first, followed after several days by a latent virus-induced leukopenia. If inoculation of the streptococcus following the virus infection was delayed, no leukocytosis developed.

On reinoculation with streptococcus several months after the primary inoculation, no significant leukocytosis developed but a marked increase in the opsonocytophagocytic index was noted very promptly. Readministration of virus to these animals seemed to suppress this index. Marked anemia of the hypochromic, microcytic type developed in the reinoculated animals, even though the plasma iron remained high. Hypertension, edema, albuminuria, red cells and urinary casts were observed in a number of monkeys reinoculated with hemolytic streptococci. The streptococci tended to persist or recur in the throats of animals thus affected.

The Thrombic Activity of a Fraction of Rabbit Plasma Globulin. By Eugene L. LOzNER and F. H. L. TAyLoR (by invitation) and Maxwell Finland, Boston, Mass.

A globulin fraction of rabbit plasma isolated by the technique of Parfentjev was shown to have a marked effect on reducing the coagulation time of both normal and hemophilic blood in vitro. This substance clotted both citrated and oxalated blood. The conversion of fibrinogen into fibrin by this substance was independent of calcium and prothrombin concentrations. This substance therefore has thrombic activity. Preliminary observations on the control of bleeding in normal and hemophilic subjects indicate that this globulin fraction has considerable hemostatic activity.

Study of the Coagulating Action of Fer-de-Lance Venom.

By George L. Kauer, Jr. and Robert M. Bird (by invitation) and PAUl Reznikoff, New York, N. Y.

A study was made of the blood-coagulating action of a fresh saline solution of crystalline "detoxified" fer-delance venom. In vitro, oxalated and heparinized human and dog blood was used; in vivo, dogs and rabbits were used.

In vitro observations revealed a more rapid clotting action on oxalated than on heparinized blood. Using "pure" fibrinogen solution, rapid conversion to fibrin occurred. Lysis of the fibrin occurred in the presence of high concentrations of venom, but this was not appreciable in lower concentrations.

Intramuscular and intravenous injections of varying venom concentrations in rabbits resulted in prolonged clotting time and reduction of fibrinogen blood levels. The intravenous injection of $0.1 \mathrm{cc} .1$ per cent venom in dogs caused the clotting time to become indefinitely prolonged with complete disappearance of prothrombin and 
of fibrinogen. In animals surviving, the clotting time, prothrombin and fibrinogen slowly returned to normal. At no time was a "positive phase" of coagulation found.

An anti-venom rabbit serum was produced by repeated subcutaneous injections of venom. This serum protected dogs from lethal doses of venom, and even from the anticoagulant action of sublethal doses. In vitro, venom mixed with this rabbit serum was unable to clot oxalated blood.

Hemoglobin Regeneration in Blood Donors. By W. M. Fowler and (by invitation) A. P. BARER, Iowa City, Ia.

Observations have been made on the rapidity of hemoglobin regeneration in 200 blood donors who have given blood for a total of 636 transfusions. The withdrawal of 500 to $600 \mathrm{cc}$. of blood caused an average drop of 2.325 grams in the blood hemoglobin. The average time necessary for the hemoglobin to return to its original level was 49.6 days with a range of 18 to 98 days. There was a definite linear relationship between the drop in hemoglobin and the recovery period in days in spite of the great individual variations. At the end of 8 weeks 74.2 per cent of the donors had regained their original hemoglobin level but the remaining 25.8 per cent required up to 15 weeks for complete recovery.

The withdrawal of $300 \mathrm{cc}$. of blood caused an average fall of 1.238 grams in the blood hemoglobin and the average recovery period was 33.3 days.

The average rate of hemoglobin production was 0.0495 gram per day in males but was somewhat slower in females, 0.040 gram per day.

Hemoglobin regeneration was no slower after subsequent donations than it had been after the first.

From these data it is apparent that a 3-month interval is advisable between blood donations unless it is established that the blood hemoglobin has returned to its original level before this time. Subsequent donations after complete recovery did not lead to a diminished rate of hemoglobin regeneration.

Leukocytosis in Patients with Psychiatric Disorders. By S. M. SMall and O. Diethel (by invitation) and A. T. Milhorat, New York, N. Y.

Leukocytosis is of frequent occurrence in patients with emotional disorders. Most of these patients show no infectious process or physical disease to account for the elevated white cell count. Briefly, the observations on 200 psychiatric patients were as follows: There was no definite correlation between the level of the white cell count and specific psychiatric disease entities. On the other hand, in certain patients with elevated white cell counts the degree of leukocytosis was often related to the intensity of the psychopathologic emotion. This relationship was consistent in the same subject but varied from patient to patient. The specific symptoms most frequently associated with leukocytosis were panic reactions, agitation, excitement with overactivity, and anxiety. Improvement in the emotional reactions, either spontaneous or induced by sedation, was associated with a return of the leukocyte count to normal levels. The intravenous administration of sodium amytal induced striking reductions in elevated leukocyte counts when there were accompanying favorable changes in the emotional status. The specific gravity of the blood plasma often varied in patients with leukocytosis, and usually was reduced by intravenous sodium amytal; however, changes in white cell counts and plasma specific gravity occurred independently of each other.

The Effect of Alterations of the Acid-base Balance Upon $\mathrm{CO}_{2}$ Output by the Lungs. By Jack D. Rosenbaum (by invitation) and JoH N P. Peters, New Haven, Conn.

Respiratory $\mathrm{CO}_{2}$ output with changing acid-base balance was studied in human subjects. During overventilation, a small $\mathrm{CO}_{2}$ loss from the extracellular fluids (ECF) is associated with a large increase in expired $\mathrm{CO}_{2}$; whereas ammonium chloride acidosis causes considerable decrease in $\mathrm{ECF} \mathrm{CO}$ content with little change in $\mathrm{CO}_{2}$ ventilation. This suggests that tissue $\mathrm{CO}_{2}$ content may change independently of alterations in total $\mathrm{CO}_{2}$ concentration of the ECF.

This hypothesis is confirmed by observations that intravenously administered bicarbonate is distributed through the ECF alone, indicating impermeability of tissue cells to $\mathrm{HCO}_{3}^{-}$ion. Molecular $\mathrm{CO}_{2}$, however, has been shown by others to diffuse through total body water. Consequently, tissue $\mathrm{CO}_{2}$ content varies with $\mathrm{CO}_{2}$ tension of the ECF, but not with $\mathrm{HCO}_{3}^{-}$ion concentration.

During overventilation, although serum total $\mathrm{CO}_{2}$ content falls but slightly, serum $\mathrm{CO}_{2}$ tension decreases markedly; large amounts of $\mathrm{CO}_{2}$ enter the blood from the tissues to be lost through the lungs. Conversely, if serum $\mathrm{CO}_{2}$ tension rises following $\mathrm{NH}_{4} \mathrm{Cl}$ ingestion, $\mathrm{CO}_{2}$ diffuses from blood to tissues and does not appear in the expired air.

Evaluation of the influence upon respiratory exchange of acid-base alterations necessitates analysis of the mechanisms of each change.

Vitamin A Metabolism in Obstructive Jaundice. By John D. Stewart and G. Margaret Rourke (introduced by Allan M. Butler), Boston, Mass.

The relationship between concentration of vitamin A and carotenoids in the plasma and vitamin $A$ in the liver has been studied. Biopsy of liver tissue has been done at operation on patients with liver disease (obstructive jaundice) and on control patients without liver disease. The patients with obstructive jaundice frequently showed zero values in plasma and liver. By giving massive doses of vitamin A parenterally before operation the hepatic content could be brought to normal, but the plasma concentration rose more slowly. The presence of normal plasma carotenoid concentration and reduced vitamin A concentration probably indicated liver damage and failure in conversion of the provitamin. Disturbances in absorption and conversion of carotene, and in storage of vitamin A, may occur in obstructive jaundice. No close correlation was found between plasma and hepatic concentrations. 
The Collection and Analysis of Fluid from Single Nephrons of the Mammalian Kidney. By ARTHUR M. Walker and (by invitation) Phyllis A. BotT, Jean Oliver and Muriel C. MacDoweli, Philadelphia, Pa., and Brooklyn, N. Y.

A technique has been developed for visualizing the surface of the mammalian kidney and for collecting fluid from single glomeruli, and proximal and distal convoluted tubules which appear there. Sufficient fluid (0.1 to 1.0 c.mm.) can be collected to permit quantitative analysis by methods which proved serviceable in a similar type of experimentation on the amphibian kidney. The site of the fluid collection is determined by macerating the kidney and isolating the punctured nephron in its entirety by microdissection.

Sixty successful experiments have been performed on rats, guinea pigs, and opossums. Glomerular fluid proves to be free from detectable amounts of protein, to have the same vapor pressure as blood plasma, and to contain glucose and exogenous creatinine in the same concentrations that exist in plasma water. The proximal tubules not only reabsorb glucose in these animals, as they do in amphibia, but also reabsorb about 80 per cent of the fluid in glomerular filtrate. This fluid reabsorption is accomplished without any increase in vapor pressure of the tubule contents but it is not a reabsorption of undifferentiated glomerular fluid, for the chloride concentration of proximal tubule fluid is half again as high as that of blood plasma. The results of the chloride analyses imply the preferential reabsorption of some other anion $\left(-\mathrm{HCO}_{3}\right.$ ?) by the proximal tubule.

Acidosis in the Premature Infant. By William SterRy Branning (introduced by Wilburt C. Davison), Durham, N. C.

Acid-base determinations done on fifteen apparently well infants whose birth weight was below 2250 grams showed that, on a diet of 120 calories per kilo per day, plasma bicarbonate was consistently lower in them than in infants of normal birth weight, and the organic acids in blood amounted to approximately three times the normal value. Total base, chloride, and phosphate all remained within the normal range.

High organic acid values in blood are supported by the fact that organic acid excretion in urine averaged $2.6 \mathrm{~m}$. eq. per kilo per day as opposed to the "normal " range of 0.6 to $1.0 \mathrm{~m}$. eq. per kilo per day for adults. All urine specimens taken had low $\mathrm{pH}$ values, averaging $\mathrm{pH}$ 5.27. The titrated total organic acids averaged $55.6 \mathrm{~mm}$. eq. per liter; of this, lactic acid composed 10 to 20 per cent, uric acid 2 to 4 per cent.

Determinations made on six infants with clinical symptoms of acidosis showed total base, chloride, and phosphate all within the normal range. Bicarbonate ranged from 9.0 to $16.0 \mathrm{~m}$. eq. per liter plasma ; calculated organic acids 11.6 to $28.0 \mathrm{~m}$. eq. per liter. Organic acid excretion varied from 22 to $100 \mathrm{~m}$. eq. per liter.

Conclusions. The organic acid constituents of plasma and urine of premature infants are usually two to three times that considered normal, and plasma bicarbonates are correspondingly low. In blood and urine, lactic acid and uric acid account for, at most, 15 to 25 per cent of the organic acid present. The premature infant is always on the borderline of acidosis.

Studies on Experimental Hypochloremia in Man. By Joseph B. KIRSNer and Kathry KNowlton (by invitation) and Walter Lincoln Palmer, Chicago, Ill.

In connection with certain studies on alkalosis previously reported, one of us (J. B. K.) produced severe hypochloremia and alkalosis without marked hyperazotemia in the dog by the daily withdrawal of gastric juice. No definite functional or anatomic evidence of renal injury was noted in these animals. The present report deals with a hypochloremia (ca. $60 \mathrm{mM}$./L.) similarly produced in man. A state of acute collapse resembling in some respects an Addisonian crisis is described. Detailed studies of the blood electrolytes disclosed such abnormal values as:

Serum Cl 54-60 mM./L. (normal 99-108)

Serum $\mathrm{CO}_{2}$ 40-44 mM./L. (normal 22-30)

Serum pH 7.60-7.70 (normal 7.35-7.48)

$\mathrm{Na}$ 115-130 mEq./L. (normal 137-147)

Total base 134-146 mEq./L. (normal 150-160)

The blood urea nitrogen and the urea clearance were not altered significantly. The volume of urine excreted was large; at times the urine contained no chloride and almost no sodium. Dehydration, as evidenced by a high red blood cell count, increased hematocrit readings, plasma protein values, and a decrease in body weight, was present despite the administration of adequate amounts of fluid. This was due apparently to the inability of the body to retain water.

The chloride content of the gastric secretion was not altered appreciably.

These studies further emphasize the importance of the electrolytes of the blood in the maintenance of normal water balance and also show that the profound alterations seen in alkalosis secondary to chloride loss are independent of demonstrable renal injury.

Studies on the Excretion of Bromsulfalein in the Bile. By A. Cantarow (introduced by Hobart A. Reimans), Philadelphia, $\mathrm{Pa}$.

After intravenous injection of bromsulfalein $(2 \mathrm{mgm}$. per kgm.), the curve of its excretion in liver bile was determined by the use of the Evelyn photoelectric colorimeter. Studies were performed on bile-fistula dogs and human subjects. Normally, the dye appeared in the bile within 15 minutes and reached a maximum concentration in 45 to 60 minutes, falling to a low level at 2 hours, and often not disappearing completely for 5 to 6 hours. Normally, 35 to 83 per cent of the quantity injected was excreted in the first hour and 61 to 100 per cent in the first 2 hours. Abnormal excretion of the dye was evidenced by one or more of the following phenomena: (1) delayed removal from the blood; (2) delayed entrance 
into the bile; (3) delayed attainment of maximum concentration; (4) prolonged high curve of excretion; (5) subnormal concentration; (6) abnormally low excretion within 1- or 2-hour periods after injection. Since 85 to 95 per cent of the dye leaves the blood within 5 minutes, this method permits practical study of two phases of bromsulfalein excretion: (1) the rate of its removal from the blood (reticulo-endothelial cells ?) and (2) the rate of its passage into the bile (hepatic cells).

Further Observations on the Oral Administration of Citrated Blood in Man. By Leon Schiff and (by invitation) R. J. Stevens, N. Shapiro, and S. Goodman, Cincinnati, $\mathrm{O}$.

Studies were carried out in a group of thirty subjects who were given varying amounts of citrated human blood (from $1 \mathrm{cc}$. to $2000 \mathrm{cc}$.) by mouth or by stomach tube. Observations were made on the following points:

1. The amount of blood necessary to produce a positive chemical test for occult blood.

2. The amount of blood necessary to produce a tarry stool.

3. The number of tarry or bloody stools, and the duration of a positive chemical test for occult blood following administration of large quantities of blood.

4. Time required for blood in the stomach to assume "coffee-grounds" appearance.

5. Relationship to nausea and vomiting.

6. Effect on temperature, white and red cell counts.

7. Effect on icteric index and urobilinogenuria.

The clinical implications of these observations are discussed.

Further Studies Concerning the Effects of Sulfanilamide on Acid-Base Metabolism. By William W. Beckman and OtTo Krayer (by invitation) and Walter Bauer, Boston, Mass.

Measurements of sodium and chloride excretion by the heart-lung-kidney preparation were made before and after the administration of sufficient sulfanilamide to produce a blood level of $20 \mathrm{mgm}$. per cent. Notwithstanding the very diminished excretion of sodium which these preparations regularly exhibit, following sulfanilamide there was a definite acceleration in the excretion of this ion. This increase was not accompanied by a similar increase in chloride excretion. Simultaneously in the serum there was a small drop in the concentration of sodium and bicarbonate and a rise in the concentration of chloride. The direction of these changes was the same as that previously described in human subjects receiving sulfanilamide. Hence these experiments define to some degree the search for the manner of production of the unusual type of acidosis encountered when sulfanilamide is given.

Reactions of Patients with Malignant Tumors to Injection of Bacterial Filtrate. By Austin M. Brues, Boston, Mass., and (by invitation), M. J. Shear, Bethesda, Md. Four patients with inoperable malignancy were given injections of that fraction of $B$. prodigiosus filtrate, pre- pared by Shear and Turner, which produces hemorrhage in and, occasionally, regressions of animal tumors, particularly sarcoma ( $c f$. Shwartzman reaction). All patients had a chill, transitory fever and leukocytosis. In one patient (multiple myeloma) the course of the disease appeared to be unaltered. In two patients (Ewing's sarcoma and metastatic carcinoma of prostate) there was immediate pain in tumor areas, followed by relief of symptoms for a time. One patient with lymphosarcoma, treated when in extremis, showed clinical evidence suggesting widespread destruction of tumors and remained well for some time. All patients died with malignancy. Two showed evidence of hemorrhage into tumors at postmortem, although the causal relation of this to filtrate injection is not certain. Two patients showing considerable relief of symptoms rapidly developed high nonprotein nitrogen (90 and $110 \mathrm{mgm}$. per cent) and uric acid (16 and $27 \mathrm{mgm}$. per cent) levels in the blood. One patient excreted, on a low purine diet, $2500 \mathrm{mgm}$. of uric acid in the first 24 hours. In two cases there was evidence of congestive failure following treatment. Successive treatments had little additional effect, even with increasing dosage. Response to waste products may be a major medical problem with any chemotherapeutic agent causing rapid destruction of tumor tissue.

The Diagnostic Value of Synovial Fluid Examination. By Marian W. Ropes and Howard C. Coggeshall (by invitation) and WAlter BAuER, Boston, Mass.

Detailed examinations of 750 synovial fluids from various types of joint disease have been made. Analyses of these data indicate that the majority of the fluids can be separated into two groups. Group I fluids are essentially traumatic in nature (traumatic arthritis, degenerative joint disease, Charcot joints and osteochondromatosis). Group II includes those obtained from specific infectious arthritides of known origin and rheumatoid arthritis.

Most Group I fluids are clear and do not clot, whereas Group II fluids may be clear or turbid and usually do clot. Total leukocyte counts above 1000 are rarely encountered in Group I fluids, whereas in Group II they are generally 3000 or higher. The polymorphonuclears are less than 500 in Group I and ordinarily greater than 1000 in Group II. The protein concentration is less than 5.2 grams per cent in Group I but varies from 3.0 to 8.9 grams in Group II. The changes in the globulin fraction are most significant. The mucin concentration is usually lower in Group II, but more important than the concentration are its precipitability with acetic acid and the viscosity of the fluid. The mucin precipitate from Group I fluids is ropey and the solution clear, while the precipitate from Group II fluids is usually friable and the solution cloudy. Group I fluids usually show only a moderate reduction of viscosity, as compared to a marked reduction in most Group II fluids. In Group I the serum-fluid fasting sugar difference is usually normal (less than $10 \mathrm{mgm}$. per cent) in contrast to a much greater difference in the majority of Group II fluids. 
To summarize, aspiration of a turbid fluid which clots and has a low viscosity, a total leukocyte count above 3000 with absolute polymorphonuclear count above 500 , a protein content above 5.0 grams per $100 \mathrm{cc}$. and a serum-fluid sugar difference of over $20 \mathrm{mgm}$. per $100 \mathrm{cc}$. indicates that the joint disease is not in Group I.

Results in individual cases have shown that examination of joint fluid used in conjunction with history, physical examination and other laboratory tests is of definite diagnostic and prognostic value.

Cytologic Study of Synovial Tissue in Various Types of Arthritis. By CURRIER McEwEN and (by invitation) Ernst W. Bergmann and Harry Most, New York, N. Y.

Synovial tissues obtained at operation have been studied by the supravital technique as well as with ordinary histologic methods. In 2 cases of relatively normal synovial tissue obtained at operation to correct traumatic disabilities, the cells found were relatively few in number and consisted chiefly of small fixed connective tissue cells. In 6 cases of osteoarthritis and traumatic arthritis of long duration in which synovial thickening was present, the cells were more numerous and were chiefly made up of fixed connective tissue cells of small to moderate size. In 6 cases of rheumatoid arthritis the cells were greatly increased in numbers and, while predominantly of moderately large fixed connective tissue type, included also mono- and multinuclear giant cells of the type previously shown to be present in the subcutaneous nodules of rheumatic fever and rheumatoid arthritis. In 13 cases of tuberculous arthritis the cells were very numerous and, while the majority of cells were of the moderately large connective tissue type found in the rheumatoid biopsies, none of the "rheumatoid giant cells" was found, but, instead, typical epithelioid cells as described by Sabin were noted.

The Distribution and Rate of Formation of Synthetized Cholesterol. By J. Roscoe MiLler and Arnold Wagner (introduced by N. C. GilberT), Chicago, Ill.

These experiments were based on the fact that deuterium cannot be incorporated into the cholesterol molecule by in vitro or in vivo procedures, but that it can be incorporated into the cholesterol molecule during the formation of that substance in the body. This offers a means of identifying the rate and distribution of cholesterol synthetized subsequent to the introduction of heavy water into the body fluids. Rats were used as experimental animals and the mass spectrograph was used for the deuterium determinations. Individual animals were sacrificed at given intervals following the introduction of heavy water and the amount of tagged cholesterol in the individual organs was determined.

Gold Metabolism and Excretion Studies in Patients Treated with Different Gold Salts. By R. H. FrEYBERG, and (by invitation) C. J. Smyth and W. D. Block, Ann Arbor, Mich.

Patients with rheumatoid arthritis were studied during and for several months following the administration of gold sodium thiomalate (myochrysine), gold sodium thiosulfate and colloidal gold sulfide. The content of gold in the blood, urine and feces was measured. The effects of different gold salts were compared.

In general, with the use of gold sodium thiomalate and gold sodium thiosulfate, the blood concentration and excretion of gold were similar. The blood content and excretion of gold increased as the weekly dose of gold increased but not in direct proportion. Results obtained with colloidal gold sulfide were exceedingly variable but with few exceptions the blood and excretion values were much smaller than those obtained with other preparations. In every instance gold was retained in the body for many months after its administration ceased; the amount retained and the length of time varied in proportion to the weekly dose of gold.

Pertinent data obtained in animal investigations of deposition and toxicity of gold are correlated.

Results are discussed in relation to the therapeutic value and the toxicity of the various gold preparations and different administration techniques, and indications will be pointed out concerning the improved use of gold salts in treatment of arthritis.

The Determination of the Relative Cerebral Metabolism in Man. By Eugene B. Ferris, JR. and (by invitation) Henry W. Ryder and Nathaniel Brower, Cincinnati, $O$.

A simple method for determining an index of the relative rate of intracranial blood flow in man has been developed. It consists in relating the rise of internal jugular venous pressure to pressure increments which are applied to the neck by means of a freely distensible cuff. The validity of the method has been tested by theoretical considerations, by checking it empirically against factors known to increase and decrease cerebral blood flow, and by comparing it with the blood flow as determined objectively by the cerebrospinal fluid displacement method. The results are reproducible in the same subject and reasonably comparable in different control subjects.

Analysis of internal jugular venous blood and of femoral arterial blood gives the arteriovenous oxygen difference. This, considered together with the "blood flow index," as obtained above, gives the relative rate of total cerebral aerobic metabolism.

In control subjects, the value for the rate of cerebral metabolism is more constant than either the blood flow or A-V difference. Illustrative examples of clinical states in which the relative total cerebral aerobic metabolism is not greatly altered and in which it is greatly reduced are presented.

There are no apparent contraindications to the procedure other than those against venous and arterial puncture and against elevating the intracranial venous pressure. 
The Clinical Significance of "Wandering" Auricular Pacemaker. By Alexander M. Burgess, Jr. (by invitation) and LAURence B. Ellis, Boston, Mass.

A study of a series of 130 patients showing so-called "shifting" or "wandering" auricular pacemaker is reported. This investigation was undertaken in order to determine the clinical significance of this electrocardiographic phenomenon which, though not uncommon, has been neglected in the literature. The cases were found to fall into two types: a "simple" type, with a cyclic shift between two distinct auricular pacemakers, and a totally irregular type with numerous foci of impulse production giving rise to contractions in no regular sequence. There were 72 cases of the "simple" type, which were found to be distributed at random among patients with and without heart disease. All 52 cases of the totally irregular type were found to have significant cardiac abnormalities, and in 90 per cent of these cases the underlying pathology was coronary artery disease. Thus it is apparent that the simple type is not necessarily of pathological significance, whereas the irregular type is an indication of heart disease. The possible mechanism of production of this phenomenon is discussed.

Observations on the Normal Mechanism of Closure of the Ductus Arteriosus. By J. Allen KenNedy and SAM L. Clark (introduced by Hugh Morgan), Nashville, Tenn.

The normal mechanism of the closure of the ductus arteriosus is not known. Our work has been concerned with an attempt to elucidate this problem by observations on the ductus arteriosus of the guinea pig fetus. Both fixed and living preparations were studied.

Normal closure of the ductus arteriosus at birth involves two phases. The first is immediate and consists of a muscular contraction requiring several minutes which obliterates the ductus lumen. The ductus when once closed (phase one) remains closed and this occurs within about 5 minutes after birth in normal animals. A second slower phase requiring several weeks involves the transformation of the obliterated muscular ductus into the fibrous ligamentum arteriosum.

Physiological closure of the ductus arteriosus can be studied in guinea pig fetuses near term. The fetuses are delivered by hysterotomy beneath a bath of warm saline with the placental vessels intact. The ductus is visualized through an opening in the chest.

The ductus will close promptly following certain stimuli. Mechanical stimuli to the ductus, such as gentle pinching or tugging, and electrical stimulation of the ductus are followed by closure in 15 to 30 seconds. Artificial inflation of the lungs with air, injection of adrenalin, massage of the carotid sinus, and hemorrhage are followed by closure of the ductus in several minutes. Under proper conditions, withdrawal of the stimulus is followed by opening of the ductus. This process of closure and opening resulting from the application and withdrawal of certain stimuli can be repeated many times.

In view of these experiments it is suggested that a patent ductus arteriosus may be due to the failure of a normal physiological mechanism rather than to a true developmental anomaly.

Circulatory Failure in Acute Infections. By RICHARD V. Ebert (by invitation) and EUGENe A. StEAd, JR. (introduced by Marshall N. Fulton), Boston, Mass.

The clinical course and hemodynamics of the circulatory failure produced by acute infections have been studied in eight patients. There were six cases of pneumococcus pneumonia, one each of streptococcus and staphylococcus septicemia. Clinically, these patients showed cold extremities, sweating, pallor, narrowing of the field of consciousness, small or absent pulsations of the radial artery, low arterial pressure, narrow pulse pressure, and tachycardia. The values for the plasma volume, the hematocrit, and the serum protein concentration, were within normal limits. The venous pressure was within normal limits before transfusion. In two patients auricular fibrillation developed after the onset of circulatory failure. Electrocardiograms failed to show any other significant variations from the normal.

The blood volume was increased by transfusions of whole blood or by administration of human plasma. Venous pressure readings were taken during the administration of the fluid, and in four cases sufficient fluid was given to increase the venous pressure. Hematocrit and serum protein determinations indicated a sustained increase in plasma volume. The arterial pressure in two cases showed a transient rise of 5 to $10 \mathrm{~mm}$. of mercury, and the radial pulse was usually palpable for a short time. There was no other evidence of clinical improvement and, although the plasma volume remained increased, the arterial pressure returned to its former level.

Although, clinically, these cases with acute infection and circulatory failure resemble cases of traumatic shock, the data indicate that the pathogenesis is different from traumatic shock, because the patients with acute infections do not show a significant degree of hemoconcentration and they are not benefited by transfusions. The fact that no improvement occurred with elevation of the lower part of the body, and the fact that little improvement occurred even when the venous return to the heart was adequate, as demonstrated by a rise in venous pressure to abnormal levels following transfusion, indicate that peripheral pooling of blood is not the primary factor in causing the circulatory failure.

The failure of the circulation to improve when the venous pressure is abnormally increased by intravenous fluids is evidence that cardiac function is not normal. The fact that the venous pressure is not elevated before transfusion, even though the cardiac function is poor, is evidence that the venous bed is not responding with normal tone to a decrease in cardiac output. The experimental evidence suggests that the circulatory failure is produced by a combination of cardiac and peripheral factors. 
The Impaired Renal Excretion of Salt in Chronic Congestive Heart Failure. By PaLmer H. Futcher and Henry A. Schroeder, New York, N. Y.

Passage of sodium chloride into the tissues in edema fluid has been regarded by many investigators as the explanation for retention of salt in chronic heart failure. The following experiments, however, suggest impairment of the ability of the kidney to excrete salt even when the blood salt level is artificially elevated above normal.

Patients maintained on a low salt diet were given intravenously 20 to 30 grams of sodium chloride as a 6 per cent solution. Further fluids were withheld for the subsequent 24 hours, during which period the serum sodium and chloride concentrations were observed to be maintained 5 to 10 milliequivalents above the upper limit of normal. The urinary output of chloride and, in some experiments, of sodium, was measured.

During the 24 hours following the injection, 2 control subjects, 1 with bronchial asthma, the other with idiopathic hypoproteinemia and edema, excreted 9.9 and 8.1 grams of chloride, expressed as sodium chloride. One patient with mild heart failure excreted 6.8 grams. Of 2 patients with severe congestive failure, 1 excreted 3.4 and 1.5 grams on different occasions, and the other 3.2 grams. One patient with severe heart iailure and diminished renal function excreted 1.8 and 1.2 grams during different tests.

Experimental Production of the Syndrome of Apparent Bundle-Branch Block With Short $P-R$ Interval. By

J. Scott Butterworth and Charles A. Poindexter (introduced by Irving S. Wright), New York, N. Y.

The electrocardiographic syndrome of apparent bundlebranch block with short $\mathrm{P}-\mathrm{R}$ interval has been reproduced in cats and dogs by the use of a special amplifier. The normal conduction system of the heart is shortcircuited through the amplifier and a ventricular asynchronism is produced, giving the appearance of bundlebranch block. By reversing the direction of the current flow in this short circuit, supraventricular tachycardias can be produced.

The method is also adaptable to the study of other problems of cardiac physiology.

A Study of the Time of Reaction of the Peripheral Blood Vessels in the Right Index Finger Tip and Right Second Toe Tip to Sensory Stimuli in Normal and Senile Subjects and Patients With Renal and Diencephalic Hypertension. By G. E. Burch, A. E. CoHn, and (by invitation) C. NeumanN, New York, N. Y.

The time of reaction of the peripheral blood vessels to sensory stimuli of constant intensity (bright light, sudden noise, pin prick, local heat, local cold and electric shock) was measured by recording the time necessary for the volume of these vessels to change on being stimulated. The stimuli were applied to the external surface of the left arm just above the elbow. The changes in volume were recorded by a sensitive pneumoplethysmograph. Measurements were accurate to 0.1 second. The indi- viduals rested in the supine position in an air-conditioned room with fingers and toes at the level of their hearts. Observations were repeated several times.

The mean reaction time of the finger tip was 3.04 seconds in normal subjects; 2.90 seconds in patients with renal and diencephalic hypertension; and 4.01 seconds in senile subjects. The mean reaction time of the blood vessels of the toe tip of the normal subjects was 3.46 seconds; 3.21 seconds in patients with hypertension; and 4.07 seconds in the senile subjects. Clearly, the reaction times of the finger and toe tips of the normal and hypertensive subjects were essentially the same, while those in the senile subjects were significantly prolonged. Reference is made to the influence of psychological states.

Capillary Blood Pressure In Man. Direct Determinations in the Digits of Subjects with Normal and Elevated Arterial Pressures. By L. W. Eichna (by invitation) and James Bordery, III, Baltimore, Md.

Capillary blood pressure was measured in the digits by the direct microinjection method (Landis). In 47 hypertensive patients the capillary blood pressure varied within the same limits as in 60 subjects with normal arterial pressure. When the capillary blood pressure was measured in the same individual at different levels of arterial pressure, no correlation between the capillary blood pressure and the arterial pressure could be demonstrated, either (1) in 3 subjects in whom temporary hypertension was induced by the injection of paredrinol sulphate, or (2) in $\mathbf{4}$ hypertensive subjects whose arterial pressure fell to normal either spontaneously or after therapeutic surgical procedures.

During reflex vasodilatation and during the local vasodilatation of reactive hyperemia, the capillary blood pressure was approximately the same in subjects with elevated and with normal arterial pressure. During the local vasodilatation induced by histamine injected intradermally, the capillary blood pressure in hypertensive subjects exceeded slightly that in subjects with normal arterial pressure.

These studies indicate that in association with arterial hypertension there is, in the digits, an increased vascular resistance which is almost entirely pre-capillary in origin, and which may be released in some measure by histamine injected locally, but not by reflex vasodilatation or reactive hyperemia. These conclusions apply only to the digital circulation.

Total Cardiac Vibrations Recorded by the Cathode Ray. By William B. Kountz and (by invitation) JoHn R. Smith, St. Louis, Mo.

Studies on cardiac vibrations over many years have embraced the limited range of audible frequencies comprising the heart sounds. An analysis of the total cardiac vibrations recorded by the cathode ray shows many variations of vibrations and also low-frequency vibrations (inaudible) which have never before been appreciated. Low frequency inaudible waves have been found in the tracing of normal persons, but are more frequently seen 
in those with heart disease, particularly those with a weakened myocardium. In the latter, the low frequency deflections may assume excessive size; in some cases such changes antedate electrocardiographic alterations.

Low frequency systolic-diastolic waves of identical character may be obtained from the experimental heart under well-controlled conditions. The physiological factors which initiate the waves are not known, but appear to be subtle motions of the myocardium itself. Under experimental conditions the waves likewise increase in size as myocardial anoxemia progresses.

It appears that large low frequency waves in vibrocardiographic tracings from patients with heart disease may be reflections of myocardial disease. These may occur before other means of examination elicit the presence of a diseased heart.

The Circulation in Traumatic Shock. By N. E. Freeman and (by invitation) M. L. Cullen and A. E. Schecter, Philadelphia, $\mathrm{Pa}$.

Traumatic shock was produced in nine of eleven dogs and in five partially sympathectomized dogs. Local fluid loss was excessive in all instances. The earliest sign of shock as studied by us was a marked reduction of peripheral blood flow as measured in the dog's paw. Hemoconcentration was not a significant finding.

Shock was produced in fourteen of sixteen dogs with more severe trauma, but with restriction of local fluid loss. The earliest sign of shock was, as a rule, a marked reduction of peripheral blood flow. There was considerable hemoconcentration in comparison to the earlier experiments.

Blood volume determinations by the carbon monoxide method showed a substantial reduction of blood volume after trauma, and this reduction was only partially accounted for by local fluid loss.

Postmortem examinations suggest that at least part of the "lost" blood volume is to be found in the lumen and mucosa of the intestinal tract.

Electrocardiographic Tracings in Normal and Abnormal Hearts Studied by the Method of Schlesinger. By P. M. Zoll and E. SpIegl (by invitation) and H. L. Blumgart, Boston, Mass.

A comparison of the electrocardiographic tracings and the postmortem findings was made in 201 cases in which complete pathologic study of the myocardium and coronary arteries was made, utilizing the Schlesinger method of injection. Measurement of the collagen content of the myocardium was also done in many instances.

Patients with normal hearts frequently showed electrocardiographic changes usually considered abnormal, such as delayed $\mathrm{A}-\mathrm{V}$ conduction, axis deviation, deviations in the $Q, S, T$ and ST intervals, notching of $Q R S$, and even negative monophasic $\mathrm{QRS}_{\mathbf{4}}$. These abnormalities were more frequently observed when the tracings were taken during acute non-cardiac illness, in which instances they may be ascribable to myocardial ischemia of non-cardiac origin. These observations indicate that the limits of the so-called "normal electrocardiogram" must be extended and that deviations now considered pathologic are compatible with the finding of a normal heart on postmortem examination.

In the absence of myocardial infarction or extensive fibrosis, patients with old coronary occlusions of the main coronary arteries may show no electrocardiographic differences from those tracings taken in patients with normal hearts. This is in accord with previous clinical and pathologic studies by us which show that the collateral circulation may compensate fully for complete occlusion of a coronary artery.

The pathologic findings of acute anterior and posterior wall infarction were closely reflected by typical and progressive electrocardiographic patterns. Single tracings, supposedly characteristic of acute infarction, were seen, however, during episodes of "acute coronary failure" (i.e., prolonged myocardial ischemia without infarction) with a rapid return to normal after the attack. ST and T wave deviations similar to those found in acute infarction were also seen in acutely ill patients with normal hearts. The fact that such changes may be witnessed secondary to disease of non-cardiac origin is of considerable importance in questions of differential diagnosis between cardiac and non-cardiac pain in seriously ill patients. Unless it is recognized that such changes may occur in the absence of cardiac disease, an erroneous diagnosis of myocardial infarction may be made.

Studies on the Effect of Cold and Heat in Patients With Angina Pectoris. By A. S. Freedberg, E. D. SpIegl (by invitation) and J. E. F. Riseman, Boston, Mass.

The increased frequency of attacks of angina in daily life when patients exercise in the cold is well recognized. This clinical fact has been utilized in the laboratory to precipitate attacks of angina at will by having the patients exercise in a room kept at a constant cold temperature (45 to $55^{\circ}$ F.). This "Standardized Exercise Tolerance Test" has been of value in diagnosis and in measuring objectively the response to treatment. This communication presents evidence concerning the manner in which cold operates to facilitate the production of angina.

Nine of a series of 20 patients with angina pectoris were able to do an appreciably greater amount of work when exercising at ordinary room temperature (75 to $80^{\circ} \mathrm{F}$.) than when exercising in the cold temperature room. When these patients exercised at room temperature, while holding an ice cube in one hand, angina was precipitated by the same minimal amount of work which induced an attack in the cold temperature room. Conversely, when these patients immersed their hands in hot water $\left(110^{\circ} \mathrm{F}.\right)$, or strapped a hot water bottle to the body before exercising in the cold temperature room, they were able to do as large an amount of work as was possible when exercising at room temperature. No appreciable differences in the heart rates, blood pressures or electrocardiograms of these patients were observed under the various conditions of temperature and exercise used in these studies. One patient receiving digitalis developed 
anoxemic changes in the electrocardiogram shortly after exposure to cold without exercise. Each of these 9 patients showed a favorable therapeutic response to vasodilating drugs.

The remaining 11 patients showed no appreciable difference in the amount of work necessary to induce angina at room temperature or in the cold. These patients with a fixed exercise tolerance showed no appreciable therapeutic response to vasodilating drugs.

To summarize, there are two groups of patients with angina on effort: (1) those in whom the exercise tolerance is unaffected by moderate changes in temperature as used in these studies; (2) those in whom the exercise tolerance is definitely decreased by cold. The reduction in exercise tolerance in a cold room may be duplicated by exercise in a warm room if the patient holds ice in his hand. These observations accord with the concept that coronary artery vasomotor changes, probably reflex in origin, exert a contributory influence in angina pectoris and correspond to the clinical observation that patients with angina pectoris, even though warmly clothed, suffer attacks particularly on cold days. These observations are likewise helpful in learning whether a patient will benefit by the prophylactic administration of vasodilator drugs.

Dynamics of Blood Flow in Thrombo-angiitis obliterans. By M. LANDOWNE* (by invitation) and L. N. Katz, $\dagger$ Chicago, Ill.

Studies in blood flow in thrombo-angiitis obliterans were made by a critically evaluated plethysmographic method. Fifty-two experiments are reported on the horizontal foot-leg of 6 ambulatory patients, 30 to 45 years of age. The recorded "initial" resting flows were compared with " maximal" resting flows; the latter being induced by heat (plethysmographic temperatures of 42 to $44^{\circ}$ C. for 30 minutes), by reactive hyperemia (cuff inflated to 200 to $300 \mathrm{~mm}$. $\mathrm{Hg}$ about the thigh for 5 to 15 minutes) and/or by both together.

Recorded "initial" resting flows showed little fluctuation. The values (1.2 to $7.5 \mathrm{cc}$. per minute per $100 \mathrm{cc}$. limb) were within the range of control observations on 18 normal subjects.

"Maximal" resting flows (2.5 to $7.5 \mathrm{cc}$. per minute per $100 \mathrm{cc}$. limb) recorded with the dilating procedures used in these patients showed no significant increases over the "initial" level, unlike controls in whom marked increases in flow were consistently demonstrated ( 7.3 to $22.6 \mathrm{cc}$. per minute per $100 \mathrm{cc}$. limb). The slight increases noted occurred in the patients with milder involvement.

Since in this condition the presumed site of disease is not in the smallest blood vessels, it is assumed that these tiny vessels are capable of being dilated if not already dilated. The results are consequently interpreted as offering a measure of the effective obstructive resistance of the diseased larger vessels, which limits the amounts of blood flowing into the limb per unit of time (for a given cardiac output and central blood pressure). This does not ex-

* Emanuel Libman Fellow.

$\dagger$ Aided by the A. D. Nast Fund for Cardiac Research. clude the possibility of change in distribution of blood within the limb following these dilating procedures.

Changes in Cardiac Output, Fluid Volume, and Kidney Function on Recovery from Congestive Heart Failure. By Walter H. Pritchard, William B. Seymour, and L. P. Longley (introduced by J. M. Hayman, Jr.), Cleveland, $\mathrm{O}$.

Cardiac output, blood volume, interstitial fluid volume, plasma proteins, etc., and inulin, urea, and phenol red clearances were determined on 7 patients which congestive heart failure, and again when maximal compensation was secured by rest, digitalis, and other therapy. Cardiac output determined by a modification of Bazett's method increased 13 to 85 per cent in 6 patients; in the seventh there was a decrease of 23 per cent at a time when he was showing evidence of digitalis intoxication. Venous pressure, blood volume (Evans Blue method) and extracellular fluid (thiocyanate method) value decreased in all cases. The change in the last of these approximated the loss of body weight. While the concentration of serum proteins rose, the total serum protein in circulation fell by 9 to 48 grams in 6 patients; loss of serum albumin accounted for approximately $2 / 3$ of the total. Phenol red clearance varied with cardiac output, as did inulin with one exception. The fraction of the cardiac output represented by the inulin clearance was quite constant. Urea clearances were more variable.

\section{The Form of the Dog's Precordial Electrocardiogram in} Bundle-Branch Block Complicated by Anterior Infarction.* By Herman Erlanger, Francis F. RosenBAUM, Nelson Cotrim (by invitation) and FrankLIN D. Johnston and Frank N. Wilson, Ann Arbor, Mich.

Dogs of medium or large size were fully anesthetized and the heart was exposed under aseptic conditions. Either the right or left branch of the His-bundle was then cut and the anterior descending branch of the left coronary artery was ligated at a high level. If the heart survived these procedures, the chest was fully restored, and the animal was allowed to recover. One to seven weeks later an extensive electrocardiographic study, including multiple precordial leads and leads from the surface of the exposed heart, was made. Of 16 animals studied in this way, 3 had right branch block without infarction; 3 had right branch block plus infarction; 1 had left branch block without infarction, and two had left branch block plus infarction. The observations made justify the following conclusions: In canine bundle-branch block, precordial leads yield curves which are similar in every respect to those obtained in human branch block of the same variety. In canine right branch block complicated by infarction, precordial leads yield QRS-complexes of characteristic form; large $Q$-waves are present in leads from points

* An experimental study carried out with the help of a grant to F. N. Wilson from the Horace H. Rackham School of Graduate Studies. 
overlying the infarcted region and late $R$-waves occur in leads from the right side of the precordium. In canine left branch block complicated by infarction, the precordial curves are difficult to distinguish from those obtained in uncomplicated left branch block.

Dorsolumbar Sympathectomy for Essential Hypertension: Results and Bearing on Etiological Considerations. By Robert Sterling Palmer and (by invitation) Reginald H. Smith wick, Boston, Mass.

A theory for the etiology of essential hypertension in man must embrace certain proved and a few probable facts :

1. Perturbation of the renal circulation by compression of the main renal arteries in experimental animals apparently alters a pressor-depressor hormonal balance, resulting in a pressor effect and systemic arterial hypertension.

2. There is no pathognomonic pathological change in the arterioles in the terminal stage of continued arterial hypertension, but there is a pathognomonic localization of such change when of marked degree, namely the renal arterioles.

3. Congenital hypoplasia, metabolic faults, infection or stases may cause the perturbation of the circulation or explain the specific localization of arteriolar disease in about 20 per cent of patients with continued arterial hypertension. In 80 per cent of patients with continued arterial hypertension there is no such explanation of the specific localization.

4. Partial or total sympathectomy does not prevent or modify experimental hypertension which follows the irreversible narrowing of the main renal arteries by application of the Goldblatt clamp, but partial sympathectomy, sufficient to denervate the kidneys, adrenals and the splanchnic bed, significantly lowers the blood pressure in a majority ( 60 per cent) of patients with so-called essential hypertension.

There appear to be only two explanations of the last fact: (a) Sympathectomy modifies the effect of organic renal constriction in man, contrary to the experience with experimental animals. Due to man's habitually upright position, according to this possibility, splanchnic denervation causes venous pooling, diminished return of blood to the heart, decreased output which lowers the head of the pressure component of arterial hypertension, and reduction of the blood pressure. (b) The alternative explanation for the favorable effect of sympathectomy in patients with continued arterial hypertension is that an overactive sympathetic vasoconstrictor outflow, or an abnormal response to a normal vasoconstrictor outflow, is interrupted, thus significantly modifying the pressor responses to posture, cold, mental stress and physiological emergencies.

The venous pooling with consequent inadequate output does not explain the favorable effect obtained in the 12 per cent of cases in which a sustained and marked improvement in the blood pressure has followed supradiaphragmatic sympathectomy (Peet) without the relative postural hypotension noted following dorsolumbar sym- pathectomy (Smithwick). The latter operation was undertaken in order to insure complete denervation. The relative postural hypotension may be a by-effect rather than a direct cause of the 60 per cent favorable results found thus far, since, as the relative postural hypotension becomes less marked, the favorable effect on the level of the blood pressure is maintained or even enhanced. We venture to suggest that it is a special condition in man, namely, his habitual upright posture, that places the circulation of the kidney at the mercy of the splanchnic bed, the function of which is the maintenance of the circulation to functioning tissue in the upper extremities, heart, lungs and especially the head. Furthermore, in the upright posture man is under the stresses of recurring emergencies. Pressor responses in certain human strains are unusually violent. May it not be that this overactive vasoconstrictor mechanism (or overactive response to a normal mechanism), operating over a long period of time in susceptible individuals, induces the specific localization in or just afferent to the nephron? This may at first be a functional vasoconstriction, leading to organic change, and finally an organic plus functional vasoconstriction. Such an etiological train of events would seem to fit more closely the observed clinical course of essential hypertension in man than the supposition of an unexplained chance localization of organic arteriolar disease, which in turn disturbs the pressor-antipressor hormonal balance in the kidneys.

Measurements of the Circulation in Dogs with an Interventricular Septal Defect. By Eugene C. EPPINGER and Robert E. Gross (introduced by C. S. Burwell), Boston, Mass.

A communication (4 to $6 \mathrm{~mm}$. in diameter) between the ventricles of the heart was produced in 5 dogs. Before establishing the communication, determinations of the cardiac output were made by the direct Fick method. After operation, the pulmonary blood flow was determined by the comparison of blood from the aorta with that from the pulmonary artery (aorta-pulmonary artery oxygen difference) and the peripheral blood flow by a comparison of the blood in the aorta with that in the right auricle (aorta-right auricle oxygen difference).

These studies showed that such a communication between the ventricles leads to:

1. A higher oxygen content in the blood of the right ventricle than in the right auricle, indicating a shunt of blood from the left to the right ventricle. This leak amounted to 30 to 50 per cent of the output of the left ventricle.

2. An increase in the amount of blood flowing through the pulmonary artery and a decrease in the peripheral blood flow.

Since the blood shunted from the left to the right ventricle promptly returns to the left ventricle via the pulmonary circulation, the output of the left ventricle continues to be the same as that of the right.

In these animals the total output of both ventricles is greater after the production of the septal defect than be- 
fore; therefore, the lesion imposes a significant burden on the heart and represents a considerable amount of wasted cardiac effort. X-rays of the heart taken before and after producing the lesion have shown that the presence of such a septal defect leads to a marked increase in the size of the right ventricle.

The following observations in one dog are cited as an example :

Cardiac output before operation .....3.15 liters per minute

After operation:

Pulmonary blood flow ........ 4.07 liters per minute

Peripheral blood flow ......... 2.63 liters per minute

Amount of shunt ........... 1.44 liters per minute

A Simple Method for the Bioassay of Renin. By Otro

Schales and Florence W. Haynes (introduced by G. P. Grabfield), Boston, Mass.

In connection with work on hypertension it was of importance to have a simple method for testing a large number of extracts for their renin content. Rabbits were used for the tests as their blood pressure is conveniently measured in the ear artery by a membrane manometer. A test procedure was standardized which, despite its simplicity, gives reliable and reproducible results. The extracts were injected intravenously in the right denervated ear. For the assay of each extract 4 animals were used and amounts were injected which would cause a rise in blood pressure of 20 to $40 \mathrm{~mm}$. mercury.

From the data on 4 animals there was calculated the amount of renin (as micrograms of nitrogen) which had to be given per kilogram of rabbit to cause an average rise in blood pressure of $30 \mathrm{~mm}$. We call this amount one rabbit unit.

If $S=$ sum of renin per kilogram (in micrograms of nitrogen) in 4 rabbits and $B=$ sum of rises in blood $S$

pressure in 4 rabbits, then $\frac{S}{B} \times 30=1$ R.U.

This method for the bioassay of renin makes it possible to give data concerning yield and degree of purity for each step of the various chemical procedures involved in the preparation of renin.

Electrocardiographic and Radiological Studies of the Heart During Normal Pregnancy. By A. GersoN Hollander (introduced by J. Hamilton Crawford), New York, N. Y.

During the course of pregnancy, especially when murmurs are present, the question arises as to whether organic heart disease is present. X-ray changes are sometimes found which seem to support such a diagnosis. In order to study the $x$-ray variations which occur in the heart during normal pregnancy, eleven cases with no suspicion of heart disease have been followed throughout pregnancy and postpartum. In addition, a series of twenty individuals who had suspicious findings during pregnancy, but who postpartum proved normal, was analyzed. Postero-anterior, left and right oblique views with the barium-filled esophagus were taken. The most common finding in both series was an invasion of the anterior wall of the esophagus, usually with no displacement of the esophagus as a whole. Another change less frequently present was prominence in the region of the pulmonary conus and pulmonary artery and, on rare occasions, elevation of the left bronchus. These changes seldom took place simultaneously.

Twenty-two normal women were followed throughout pregnancy with serial electrocardiograms, leads I and III being taken synchronously. The common alterations which took place were the development of a deep $Q$ wave and a negative $T$ in lead III. There was a gradual shift of the electrical axis to the left of 20 to 30 degrees. The axis, however, at no time exceeded the recognized normal limits.

A Piezoelectric Manometer for Various Clinical Studies of the Form and Velocity of the Intraesophageal Pressure Pulses. By JAN NyBoER (introduced by Herman O. Mosenthal), New York, N. Y.

A successful attempt was made to substitute an electrical method (J. Clin. Invest., 1938, 18, 511) for the conventional mechanical one of an optical membranemanometer as the terminal pressure instrument for intraesophageal pulse studies. In this device, a small rubber balloon was connected by a (Number $16 \mathrm{Fr}$.) soft rubber esophageal catheter to a metallic aneroid on which was mounted a wire leading to a piezo-crystal unit. The potentials generated from the crystal by the undulating pressures in the aneroid were recorded by a string galvanometer controlled by a vacuum-tube amplifier. Simultaneous tracings of pressures by both methods proved an equivalent accuracy but greater convenience and increased sensitivity of the electrical device was noted as compared with the mechanical one.

For purposes of study of the relative velocity and direction of the complicated pressure changes during the cardiac cycle, the piezoelectric potentials may be electrically differentiated by the method described elsewhere ( $\mathrm{J}$. Clin. Invest., 1940, 19, 963).

For increased accuracy in reference to a given retrocardiac level, simultaneous esophageal electrocardiograms were made from an electrode fixed at the tip of the catheter near the esophageal balloon. Tracings were taken in $2.5 \mathrm{~cm}$. steps between the lower end of the esophagus and a position opposite the aortic arch. These pressure pulses confirm in part their recent description by Taquini (Am. Heart J., 1940, 20, 129) and suggest their probable usefulness in the study of the normal and abnormal heart. An attempt was made to evaluate the juxta-ventricular esophageal pulsation in cases of posterior myocardial infarction.

The Production of Hypothyroidism in Rabbits by Injections of Thyroglobulin. Its Relation to Refractoriness. By J. Lerman, Boston, Mass.

The demonstration of thyroglobulin antibodies by Hektoen and Schulhof (J. A. M. A., 1923, 80, 386) 
raised the question, "Are these antibodies antimetabolic (antihormones)?" In 5 out of 7 rabbits immunized with human thyroglobulin there developed some degree of refractoriness to subsequent intraperitoneal injections of thyroglobulin. The negative results of other investigators (Schulhof, Am. J. Physiol., 1930, 93, 175 and Rosen and Marine, Am. J. Physiol., 1937, 120, 121) are, I believe, due to technical difficulties.

Another important attribute of thyroglobulin antibodies is that they are "hormone-specific" rather than "speciesspecific."

These two effects should theoretically lead to inhibition of the immunized animal's own thyroid. In fact, 7 of 8 rabbits immunized with human thyroglobulin over a long period of time (6 months or more) developed metabolic rates of minus 20 to minus 35 per cent below normal; they behaved in other respects like myxedematous animals. Another animal reached a level of minus 14 after 5 months of immunization. The findings of Morgan and Ivy (Proc. Soc. Exper. Biol. and Med., 1934, 31, 1139) are, in some respects, similar. They produced "cretin-like" rabbits by injection of serum from chickens which had been immunized with a crude extract of thyroid.

Inasmuch as one of our main interests is the treatment of human hyperthyroidism, and since the above results suggested a possible mode of controlling the disease, we immunized one patient suffering from Graves' disease with sheep thyroglobulin for 3 months. It was impossible to obtain a high antibody titer in the serum of this patient, and the clinical results were entirely negative. Further trials of this sort are indicated.

Pitressin in Oil. Prolonged Antidiuretic Effect in Experimental and Clinical Diabetes Insipidus. By D. J. STEPHENS, Rochester, N. Y.

The recent literature contains references to prolongation of the antidiuretic effect of posterior pituitary extracts emulsified in oil and beeswax by the subcutaneous implantation of posterior pituitary powder, by the injection of pituitrin and posterior pituitary extracts in the presence of zinc salts and by the administration of a suspension of pitressin in oil. This communication reports the results of a study of the effect of pitressin in oil in two dogs with experimental diabetes insipidus and in 7 patients with diabetes insipidus. Urine was collected in 4-hour periods and the volume, specific gravity, and, in some instances, chloride concentration were determined before and after the subcutaneous administration of an aqueous solution of pitressin and of a suspension of pitressin tannate in peanut oil. The duration of the antidiuretic effect of the largest tolerated doses of aqueous pitressin (10 to 20 pressor units) was from 4 to 8 hours. Single doses of 1 cc. of pitressin in oil (4 pressor units) exhibited antidiuretic effect of from 24 to 96 hours in duration. The maximum effect of pitressin in oil on urine volume and specific gravity was greater than the maximum effect of the largest tolerated doses of pitressin in aqueous solution. No local or general reac- tions were encountered after the administration of pitressin in oil.

Considering both intensity and duration of antidiuretic effect, pitressin in oil is at least $\mathbf{1 5}$ times as effective as aqueous pitressin. In patients with diabetes insipidus, not only is the number of hypodermic injections greatly reduced, but symptoms, urine volume, and specific gravity are more consistently and satisfactorily controlled by pitressin in oil than by any other method of treatment available at the present time.

Clinical and Biochemical Studies of Female Hirsutism and Virilism with Spccial Reference to Differential Diagnostic and Prognostic Significance of Urinary Ketosteroid Excretion. By HARRY B. FrIEdgood (introduced by James P. O'Hare), Boston, Mass.

Clinical and biochemical observations were made on 10 normal women, on 65 patients with various types of hirsutism and virilism not attributable to neoplasm, and on 2 cases of virilism due to malignant corticoadrenal tumor. Included among the clinical studies were the following: (1) Precipitating factors related to the onset of hypertrichosis (e.g., menarche, menopause, pregnancy, amenorrhoea, etc.); (2) course of development, extent and distribution of various types of hypertrichosis; (3) physiological significance of therapeutically-induced and spontaneous improvement in hypertrichosis.

Biochemical studies were concerned with establishing the range of 24-hour ketosteroid excretion in various circumstances. The ketosteroid output of normal women and of hirsute patients fell within the same range; that of virilistic women was generally outside the upper limits of normal, although these values overlapped occasionally. Ketosteroid determinations gave reliable help in the differential diagnosis between virilism due to corticoadrenal tumor and that not attributable to neoplasm. Quantitation of the ketosteroids was also of aid in determining prognosis, inasmuch as it led to the prediction of postoperative recurrence of a corticoadrenal tumor several months before this was suspected clinically.

The marked increase in urinary ketosteroids which is characteristic of tumor-induced virilism is due to the excretion of abnormally large amounts of dehydroisoandrosterone, which ordinarily constitutes only a small fraction of the androgen content of urine. Dehydroisoandrosterone is determined quantitatively, therefore, for its diagnostic value whenever corticoadrenal tumor is the suspected cause of virilism.

The Relation of Dark Adaptation (Determined by the Hecht Adaptometer) to Blood Carotene and Blood Vitamin A. Levels. By E. White Patton and W. R. Sutron (by invitation) and JoHn B. Youmans.

Using the Hecht adaptometer, the rod threshold for light perception was determined in approximately 50 individuals. In each individual, the threshold was obtained both with and without previous light adaptation. When light adaptation preceded dark adaptation, the technic described by Hecht was used. When no previous light 
adaptation was employed, the subject was allowed to dark adapt for 20 minutes and the threshold was then measured. The correlation of the rod thresholds obtained by the two technics was quite satisfactory. The group included individuals with normal and elevated thresholds, and the individuals varied in age from 8 to 72 years.

Using the threshold obtained without previous light adaptation, a comparison was made between the rod threshold, the blood serum vitamin $A$, and the serum carotene levels in approximately 400 individuals. Each subject was examined twice, once in the fall and again in the spring. The average dietary intake of the entire group was decidedly more adequate in vitamin $A$ and carotene at the time of the fall examination. Considering the entire group, little direct correlation could be discerned between the rod threshold levels and the blood serum vitamin A or carotene levels. There was a striking difference in the incidence of abnormal serum vitamin $A$ and serum carotene levels in the two examination periods. On examination in the spring, the level of serum vitamin $\mathrm{A}$ and carotene in the blood was low in a large number of the individuals. When the same individuals were reexamined in the fall, the majority of them were found to have normal levels. No significant difference was found in the number of abnormal adaptometer thresholds occurring in the two examination periods.

Further work is now in progress to evaluate the relationship of the dietary intake of vitamin $\mathrm{A}$ and carotene to these procedures, and other factors such as age, etc., which may influence them are being studied.

Hormonal Factors Influencing Skeletal Growth. By Willard O. Thompson, and (by invitation) NorRis J. Heckel and Richard M. Morris, Chicago, Ill.

Studies in man during the past several years appear to support the following conclusions:

1. The growth and configuration of the skeleton are under hormonal control.

2. The thyroid and the pituitary appear to affect primarily the length of the skeleton. There is some evidence that the thyroid affects the growth of bone by stimulating the production of growth principle in the anterior lobe of the pituitary.

3. The gonads through their production of sex hormones affect the proportions of the various parts of the skeleton, as well as its length. Thus it may be shown that :

(a) In men and women whose gonads have not functioned during the period of skeletal growth the trunk is usually very short in proportion to the extremities, the total height of the body being within normal limits. This disproportion may be in part the result of failure of epiphyseal growth in the vertebrae which occurs at the time of puberty.

(b) In young boys the administration of either chorionic gonadotropin or male sex hormone will not only cause stimulation of genital growth but will also cause the skeleton to grow in length at an abnormally rapid rate and will cause the trunk to become long in proportion to the extremities. Whether or not premature stimulation of genital growth causes premature closure of epiphyses is still a debatable point. It would appear that closure of epiphyses is dependent upon a certain amount of aging in the bone itself.

(c) In pituitary dwarfs with hypogonadism in whom the epiphyses are open, administration of the male or female sex hormone, depending upon the sex of the individual, may result in a great increase in length of the skeleton and a proportionately greater increase in the length of the trunk than of the extremities.

4. The influence of hormones is modified by hereditary and nutritional factors which affect bone directly.

The growth of the skeleton must, therefore, be thought of as a complex process which involves a delicate balance between hormonal, hereditary and nutritional factors.

Studies of Lymph in the Adrenalectomized Dog. By Oliver Cope, Adpison G. Brenizer, Jr. (by invitation), Hugo Polderman (by invitation) and Henry $K$. Bezcher, Boston, Mass.

Using local procaine anesthesia, the cervical lymphatic trunks were cannulized in trained normal and adrenalectomized dogs in various stages of insufficiency. The flow of lymph was stimulated either by rhythmic motion of the head or gentle massage. The lymph was compared with the serum of blood samples removed during collection; the proteins, amylase activity, NPN, $\mathrm{Na}, \mathrm{K}, \mathrm{Ca}$, total base, $\mathrm{Cl}, \mathrm{CO}_{2}, \mathrm{PO}_{4}, \mathrm{pH}$, sugar and hematocrit were measured. The arterial blood pressure and plasma and extracellular fluid volumes were determined.

The outstanding change in lymph encountered was a rise in protein from 2.0 to 2.5 per cent in the normal to 2.7 to 5.0 per cent in the insufficient dogs. The serum protein did not show a corresponding rise. The amylase activity * of lymph rose parallel to the activity of serum but was lower in lymph than in serum in both the normal and insufficient animal. The electrolytes, NPN and sugar followed closely the levels in the blood of both the normal and insufficient animal. The volume of lymph collected was increased in the insufficient dog. The reported de.creased plasma and increased extracellular fluid volumes were confirmed. The findings suggest increased capillary permeability in adrenal cortical insufficiency in the dog.

Measurements of the Velocity of Sound in the Human Body and the Value of this Quantity in the Design of Stethoscopes. By Franklin D. Johnston and Paul S. BArker, Ann Arbor, Mich.

When a sound wave passes from a dense into a rare

* Cope, Oliver, Kapnick, Israel, Lambert, Adrian, Pratt, T. Dennie, and Verlot, Max G. Endocrine function and amylase activity. II. Changes in activity of blood serum amylase in response to changes in adrenal cortical function in the dog and rabbit. Endocrinology, 1939, 25, 236. 
medium much of the energy in the wave is reflected at the boundary so that very little is transmitted to an observer in the rare medium (air). The physical characteristics of the two media will determine the efficiency with which such transmission takes place. Thus, sounds arising in water are very poorly heard in air since only 0.12 per cent of the energy in the wave striking the water surface passes into the air. Similar considerations apply to sounds originating within the human body.

A method for estimating the average velocity of transmission of sound through the human chest has been developed and, when this velocity is multiplied by the mean density of the thoracic structures, a very useful quantitythe specific acoustic resistance-is obtained. Knowing this, it can be shown that less than 1 per cent of the sound energy arising inside the body passes into the air.

A stethoscope serves to increase greatly this transfer of energy by acting as an acoustic matching transformer between the body and the air, and the form of the endpiece necessary to achieve this end most efficiently can be calculated from the data at hand.

The Oxygen Consumption of the Intact Mammalian Heart. By G. V. LeRoy (introduced by Richard B. Capps), Chicago, Ill.

The oxygen consumption of the heart of the intact dogs was studied by means of a modification of the direct method of Harrison, Resnik and Friedman. Two types of anesthesia were employed-nembutal and dial. The data are compared with those of Harrison, et al., who used "appropriate amounts" of morphine. In general, in this type of experiment the oxygen consumption and the mechanical efficiency of the heart vary inversely as the depth of the anesthesia. The dogs of Harrison, et al., who were not submitted to a surgical procedure displayed the highest values of oxygen consumption and efficiency; the next highest values were in our nembutal group; the next highest were found in the dogs of Harrison, et al., who had been operated upon; and the lowest values were from our dial group. Values for these same factors obtained from heart-lung preparations are generally lower because of the trauma encountered. Thus, anesthetic agents demonstrably influence cardiac energetics in an unfavorable manner.

An observation of considerable importance, and one not emphasized heretofore, is the low oxygen saturation of coronary venous blood. This value averaged 20 per cent of arterial in our series, and was lower than one had imagined it to be. A simple calculation will demonstrate that even a normal heart cannot long tolerate a reduction of the coronary flow by more than 20 per cent without suffering myocardial ischemia. 